\title{
L'économie des sensations dans la clinique hippocratique
}

Laurent Ayache

\section{(2) OpenEdition}

1 Journals

Édition électronique

URL : https://journals.openedition.org/philosant/930

DOI : 10.4000/philosant.930

ISSN : 2648-2789

Éditeur

Éditions Vrin

\section{Édition imprimée}

Date de publication : 1 novembre 2012

Pagination : 5-42

ISBN : 978-2-7574-0400-3

ISSN : 1634-4561

\section{Référence électronique}

Laurent Ayache, «L'économie des sensations dans la clinique hippocratique », Philosophie antique [En ligne], 12 | 2012, mis en ligne le 01 novembre 2018, consulté le 03 décembre 2022. URL : http:// journals.openedition.org/philosant/930; DOI : https://doi.org/10.4000/philosant.930

\section{(c) $(1) \&$}

Creative Commons - Attribution - Pas d'Utilisation Commerciale - Pas de Modification 4.0 International - CC BY-NC-ND 4.0

https://creativecommons.org/licenses/by-nc-nd/4.0/ 


\title{
L'ÉCONOMIE DES SENSATIONS DANS LA CLINIQUE HIPPOCRATIQUE
}

\author{
Laurent AYACHE \\ Lycée Dumont d'Urville, Toulon
}

RÉSUMÉ. Qu'est-ce qu'une sensation pour un médecin hippocratique ? Cette question est traitée ici à partir d'une recherche sur le rôle que les traités chirurgicaux et le Pronostic accordent aux sensations du malade d'une part, du praticien d'autre part, dans l'élaboration du jugement médical, selon ses deux moments principaux que sont l'estimation d'un pronostic et le choix d'une conduite thérapeutique. L'hypothèse mise en œuvre est celle de la réflexivité de la médecine hippocratique : la représentation que les médecins se faisaient de l'élaboration du jugement médical à partir des signes sensibles doit être conforme aux théories médicales de la sensation et du jugement. Si cette hypothèse est valide, la pratique clinique hippocratique doit refléter les conceptions médicales de la sensation et du jugement, et peut donc en témoigner. Cette étude confirme cette hypothèse et établit que le jugement résulte, pour les médecins hippocratiques, d'un mélange des sensations. Ces dernières sont comprises comme des processus physiologiques dont les vecteurs de diffusion sont, selon notamment certains textes anatomiques, les vaisseaux. Aucun texte hippocratique, pas même Maladie sacrée, ne localise un siège de la sensation ou du jugement. Les conseils adressés aux médecins pour viser l'exactitude dans un jugement toujours singulier (Ancienne médecine, 9) relèvent d'un régime des sensations («l'économie autour du malade» d'Épidémies $V I$ ) analogue au régime des aliments et des exercices que les médecins prescrivent à ceux qui les consultent. Par-delà leurs différences doctrinales, une certaine unité des traités qui forment le noyau de la Collection hippocratique est ainsi confirmée. Cette unité est d'abord celle de la théorie du mélange compris comme crase, qui joue dans la conception du jugement médical à partir des sensations un rôle éminent.

SUMMARY. To a Hippocratic mind, what is a sensation? This paper aims at addressing this question by examining which role the treatises about surgery and the Prognostikon assign to the sensations either of the patient or of the practitioner, in the working out of a prognosis and the choice of the treatment. The paper bears out the hypothesis according to which the clinical practice of Hippocratic physicians mirrored their views of sensation and judgment, and so gives evidence for them. In their view, 
judgment is the result of a mixing of sensations, that in turn are physiological processus spread through vessels : no Hippocratic text, including The Sacred Disease, locates a seat of sensation or judgment. Pieces of advice that aim at helping physicians to form exact judgments (Ancient Medicine, 9) belong to a regulation of sensations similar to the regulation of food and exercises prescribed for their patients. So, beyond their doctrinal differences, the treatises that constitute the core of the Hippocratic Collection show some sort of unity, primarily in understanding mixture as crasis. This notion plays a major role in the view of medical judgment as resulting from sensations. 
Aucun texte de la Collection hippocratique, pas même le court traité $D e$ la vision, ne présente, pour elle-même, une théorie physiologique de la sensation. Les passages qui concernent sensation ou perception sont insérés dans des développements qui relèvent de trois registres : un registre anatomique, notamment à l'occasion de la description des vaisseaux qui passent par les organes sensoriels; un registre pathologique, les troubles de la sensation (surdité, cécité, nyctalopie, etc.) apparaissant souvent comme des accidents au cours des maladies; enfin, un registre méthodologique, la sensation du malade et les perceptions du médecin étant présentées comme des guides pour le jugement médical dans le pronostic et la conduite de la thérapeutique.

Cette étude se concentrera sur la question particulière de l'usage des perceptions dans l'activité clinique du médecin, c'est-à-dire sur le registre méthodologique. Je chercherai à dégager les modalités du recours aux sensations dans l'élaboration du jugement médical. Ce faisant, j'userai indifféremment des termes « sensation » et «perception », en les comprenant dans toute l'étendue du sens que les textes hippocratiques imposent, sans m'enfermer dans une définition préalable de l'al゙ $\theta \eta \sigma ı \zeta$. Mon étude se concentrera sur quelques traités qui possèdent une certaine homogénéité, essentiellement, les traités chirurgicaux et le Pronostic.

Le recours à la sensation lors de l'examen clinique apparaît sous deux formes différentes : tantôt le médecin s'informe des sensations du malade, tantôt il recourt à ses propres perceptions. Il convient donc d'étudier ces deux usages.

\section{Le recours à la sensation du patient}

Les traités chirurgicaux font régulièrement référence à la sensation du blessé pour régler l'activité thérapeutique du médecin. Ainsi, le traité Des fractures expose la technique du bandage d'un membre cassé. L'interrogation du patient sur ce qu'il ressent sert en premier lieu à ajuster le bandage à sa conformation individuelle :

Voici les signes d'un beau traitement et d'un bandage correct (öp $\theta \tilde{\omega} \varsigma)$ : si vous demandez au blessé s'il est comprimé, et s'il dit qu'il est comprimé, 
mais modérément ( $\dot{\eta} \sigma \dot{\gamma} \chi \omega \varsigma)$, et s'il dit : « surtout à l'endroit de la fracture $(\mu \dot{\alpha} \lambda \iota \sigma \tau \alpha[\ldots] \kappa \alpha \tau \dot{\alpha} \tau \dot{o} \kappa \dot{\alpha} \tau \eta \gamma \mu \alpha) \gg\left(\right.$ c. 5 : Kühlewein, II, $53=$ III, 432 L.) ${ }^{1}$.

Par la suite, la force de la compression est adaptée, lors de chaque nouveau pansement, à l'évolution de la blessure. Le médecin est alors attentif à l'expression précise de l'évolution des sensations du blessé :

Interrogé, le blessé répondra qu'il est serré un peu plus qu'auparavant

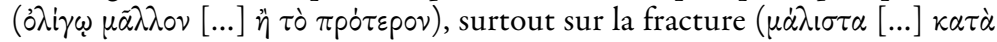
$\tau \grave{~} \kappa \dot{\alpha} \tau \eta \gamma \mu \alpha$ ), et sur le reste proportionnellement ( $\kappa \alpha \tau \dot{\alpha} \lambda \dot{o} \gamma \circ \nu)$ (ibid. : Kühlewein II, 54-56 = III, 436 L.).

L'interrogation du blessé et l'écoute attentive de ses réponses sert également à corriger l'application du bandage pour approcher la compression optimale :

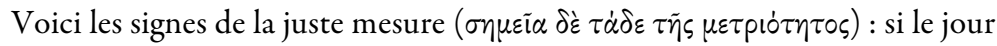
du pansement et la nuit suivante il se sent serré, non pas moins, mais davan-

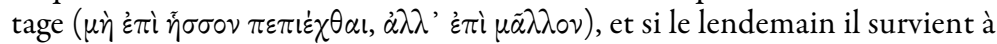
la main un peu de tuméfaction molle; tel est le signe d'une juste mesure de

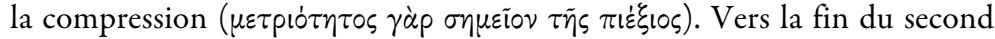
jour il doit se sentir moins serré ( $\dot{\varepsilon} \pi \grave{\eta} \sigma \sigma \circ \nu$ ), et le troisième vous devez trouver l'appareil relâché. Si quelqu'un des caractères ici énumérés est en défaut, il faut savoir que le bandage est plus lâche que la mesure ( $\chi \alpha \lambda \alpha \rho \omega \tau \dot{\varepsilon} p \eta \dot{\varepsilon} \sigma \tau i \nu$

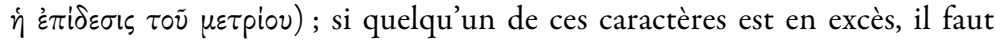

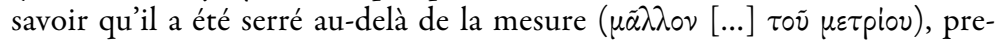

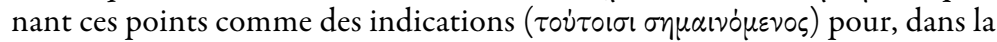
réapplication du bandage, davantage lâcher ou serrer (ibid. : Kühlewein II, $53=$ III, 432 L.).

L'évolution de la sensation du patient concourt ainsi à conduire le

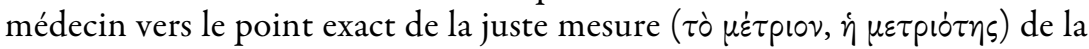
thérapeutique. Le jugement du médecin épouse l'évolution de la sensation du blessé et ajuste l'action en conséquence. Il n'applique pas uniformément une règle universelle, mais se conforme à une méthode de rectification régulièrement renouvelée dont le guide principal est la sensation du patient.

Le recours à la sensibilité du patient dépasse parfois ce rôle d'adaptation des principes conceptuels à la diversité des cas et de leur évolution. Au début de ce même traité Des fractures, l'auteur opère une véritable substitution d'une idée par la sensation du patient. Dans ce passage, l'idée produite par la théorie sert seulement de lieu commun, amorçant une démarche dont l'aboutissement est purement sensible.

1. J'indique la pagination dans l'édition utilisée (ici, Kühlewein 1895-1902) et dans l'édition Littré 1839-1861. Lorsqu'il n'existe pas de traduction française plus récente, je suis la traduction de Littré en la corrigeant le cas échéant. 
Ce traité s'ouvre en effet sur la question de l'attitude dans laquelle il faut immobiliser un bras luxé ou fracturé. La réponse théorique est qu'il faut placer le bras dans sa conformation naturelle, $\kappa \alpha \tau \dot{\alpha} \phi \dot{v} \sigma \iota v$. La théorie apporte même une détermination de cette conformation : l'attitude natu-

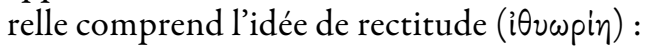

Le médecin doit, pour les luxations et les fractures, faire, autant qu'il est possible, les extensions dans l'attitude la plus rectiligne : c'est la conforma-

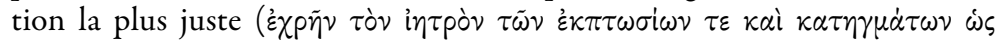

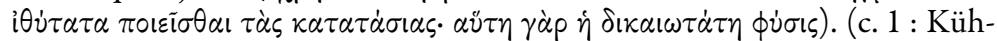
lewein II, 46 = III, 412 L.).

Et pourtant, la recherche de cette position naturelle par le raisonnement est condamnée par l'auteur du traité hippocratique : « Les médecins qui ratiocinent sont alors ceux qui se trompent (oi $\delta \dot{\varepsilon}$ in $\tau$ poi $\sigma \circ \phi i \zeta o ́ \mu \varepsilon v o l ~ \delta \tilde{\eta} \theta \varepsilon \nu$

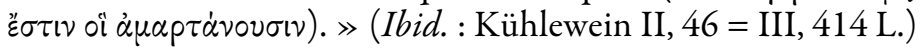

Ainsi, la détermination théorique de l'attitude naturelle conduit à des résultats aberrants :

Un blessé $[\ldots]$ présenta le bras dans la pronation au médecin qui devait le panser ; celui-ci le força à tenir cette partie comme les archers la tiennent quand ils avancent l'épaule, et il y appliqua le bandage dans cette position, considérant que c'était pour le bras l'attitude naturelle ( $\tau \dot{c} \kappa \alpha \tau \dot{\alpha} \phi \dot{v} \sigma \iota \nu)$; il alléguait en preuve ( $\mu \alpha \rho \tau \dot{p} ı$ เo $̇ \pi \dot{\gamma} \gamma \varepsilon \tau 0)$ tous les os de l'avant-bras qui sont

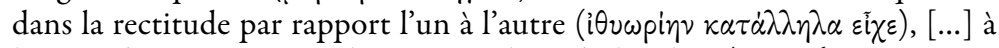
l'appui de son opinion, il invoquait l'art de l'archer ( $\tau \dot{\eta} \nu \tau 0 \xi$

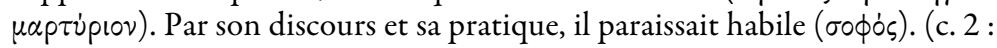
Kühlewein II, 47 = III, 418 L.)

Un autre médecin, mettant le bras dans la supination, ordonnait de faire ainsi l'extension, et il bandait le bras du blessé dans cette position,

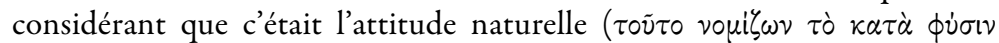

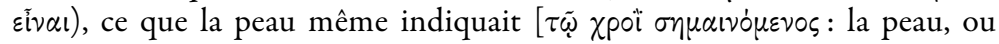
peut-être la couleur qu'elle prend dans cette attitude]; et considérant

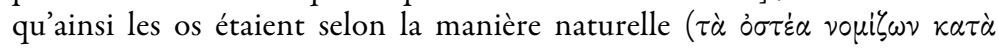

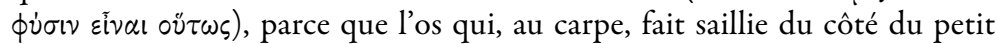

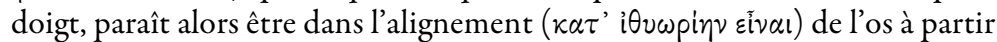
duquel on mesure la coudée. Voilà les raisons qu'il alléguait $(\tau \alpha \tilde{\nu} \tau \alpha \tau \dot{\alpha}$

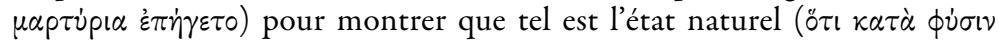

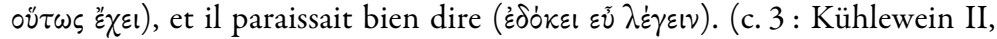
$49=$ III, 422-424 L.)

Ainsi, la détermination théorique de l'attitude naturelle par le critère du concept de rectitude n'offre pas de solution unique. Au contraire, le concept est non seulement impuissant à rassembler la diversité, mais il est confronté à une véritable explosion de singularités ; au bandage à la manière de

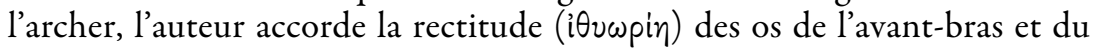


bras dans cette position « comme si le membre entier ne faisait qu'un ( $\omega s$

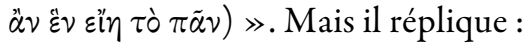

[Ce médecin] oubliait les autres arts et ce qui se fait par la force comme ce qui se fait par l'adresse, ne sachant pas que l'attitude naturelle est différente

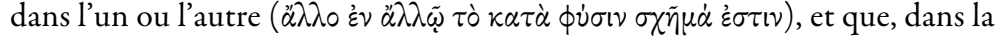
même action, il peut arriver qu'autre soit la position naturelle du bras droit

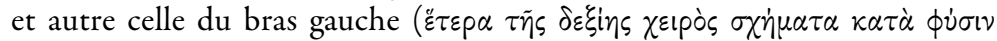

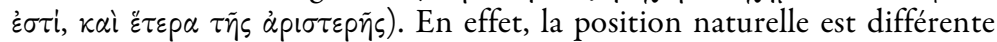
pour lancer un javelot, différente pour tourner une fronde, différente pour jeter une pierre, différente dans le pugilat, différente dans le repos. Quel que soit le nombre d'arts qu'on chercherait, l'attitude naturelle des bras

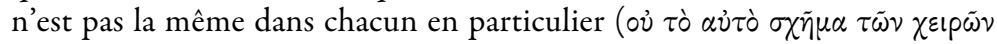

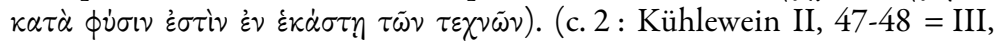
418-420 L.)

Comment dès lors déterminer la position naturelle du membre si le concept de rectitude est impuissant à sélectionner une attitude et à éliminer les autres ? Le traité De l'officine du médecin, chap. 15, corrige cette première approximation en distinguant l'attitude naturelle dans l'action et l'attitude naturelle dans le repos, puis en ajoutant la considération de l'attitude

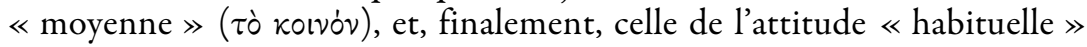

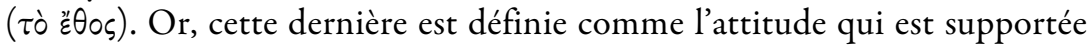
le plus facilement et le plus longtemps par le membre pansé. C'est donc, en définitive, la sensibilité du membre qui, en dernière instance, détermine le choix du médecin. Le traité Des fractures préconise ainsi une position qui, durant tout le temps de la consolidation, occasionne le moins de douleur. Ce critère sensible est tour à tour opposé aux deux positions avancées par les adversaires de l'auteur :

1. contre la position de l'archer: $\mathrm{Si}$, après avoir placé l'appareil, le médecin ordonnait au blessé de garder ainsi le bras, il causerait beaucoup d'autres

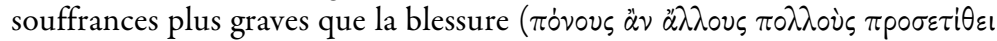

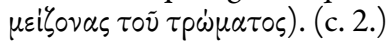

2. contre la supination: Si le bras demeurait étendu dans la supination, cela

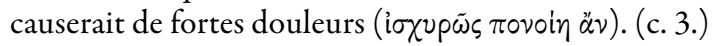

En revanche,

si l'on fait l'extension du bras cassé telle que je la recommande, [...] le bras,

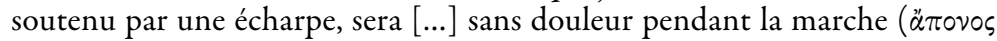

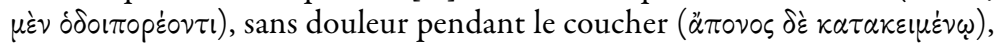
et sans fatigue ( $\dot{\alpha} \kappa \dot{\alpha} \mu \alpha \tau \circ \varsigma)$ (ibid.).

La position naturelle ne peut donc être complètement déterminée par le raisonnement. En définitive, le meilleur moyen de trouver la position la moins douloureuse pour le blessé est de le laisser présenter lui-même son bras. 
L'idée de «position naturelle » et sa détermination par la rectitude jouent dans ce passage le rôle d'une amorce pour le jugement. L'auteur ne la conteste pas, mais montre que, lors de son application sur des cas concrets, le raisonnement est impuissant à en déduire ce qui convient pour chaque cas, parce que le naturel est surdéterminé par une somme indéfinie de facteurs : non seulement la conformation des os, mais aussi les actions habituelles que le patient impose à son membre, lesquelles, peu à peu, lui impriment une attitude propre, différente selon les activités pratiquées. La sensation du patient condense en elle cette somme que le raisonnement est incapable de déployer. Le blessé ressent ainsi immédiatement ce que le médecin ne peut déduire. C'est pourquoi à l'idée de naturel se substitue le critère sensible du minimum de douleur.

Ce procédé qui passe de l'idée à la sensation par substitution plutôt que par subsomption n'annule pas le rôle de l'idée. Il lui confère la fonction d'un lieu commun - toute déligation doit respecter l'attitude naturelle du membre - qui fonde le jugement médical et conduit le médecin à chercher cette attitude en quittant le registre du raisonnement, débordé par la multiplicité des cas, pour se tourner vers ce que le patient ressent.

Si la sensation du patient peut ainsi donner congé à l'idée, c'est qu'elle est plus riche qu'un simple donné. Ainsi, lorsque, exprimant une altération du corps, la sensation devient douleur, elle indique par là même, a contrario, une norme. Elle équivaut alors à un jugement immédiat, par le corps luimême, qui refuse spontanément, dans la douleur, l'altération pathologique, comme il approuve, dans le bien-être, la santé. L'approbation et le refus ne relèvent donc pas d'un jugement sur les sensations, mais bien de la sensibilité elle-même, dans sa dimension nociceptive.

Le médecin accueille dès lors la sensation douloureuse, non comme un simple donné, mais déjà comme une prescription. C'est pourquoi la recherche du minimum de douleur peut servir de guide pour approcher cet état naturel différent selon les individus et les localisations des blessures, et évoluant au cours du temps. L'évitement de la douleur dans la déligation ne peut donc pas se résumer à un effet de la gracieuseté du médecin hippocratique. Il s'agit moins du confort du patient que de la quête, par le médecin, de la position naturelle des parties à bander, position impossible à déduire par le raisonnement mais que le patient présente spontanément parce que son corps, de lui-même, résiste aux attitudes qui suscitent de la souffrance.

\section{Critique de la sensation du patient}

Toutefois, le médecin doit soumettre cette sensation-jugement du corps malade à un examen critique. En effet, la sensibilité peut tromper, comme le montre ce passage de l'Usage des liquides : 


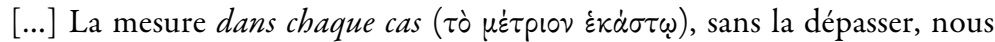
savons la discerner, comme le chaud pour la peau, par l'excès dans les deux sens ( $\dot{\xi} \xi \dot{\nu} \pi \varepsilon \rho \beta 0 \lambda \eta \tilde{s} \xi \dot{\varepsilon} \phi$ ' $\dot{\alpha} \dot{\alpha} \tau \varepsilon \rho \alpha)$; pour ne pas commettre d'erreur dans un sens ou dans l'autre, on est renseigné par le dommage ou le manque d'utilité ( tiède ; il faut en effet profiter des dommages ou des bienfaits, quand il s'en

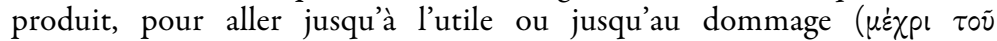

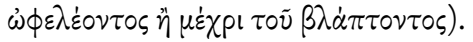

L'humectation a peu de force; le refroidissement et l'échauffement en ont beaucoup, comme par le soleil ; l'eau froide, échauffée comme pour une boisson, convient au malade faible; mais l'eau chaude ne doit pas aller jus-

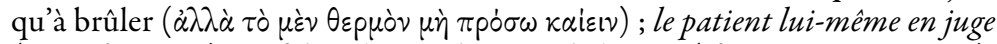

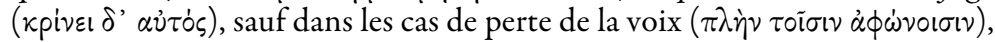
de paralysie $(\pi \alpha \rho \alpha \pi \lambda \eta \gamma$ เкоĩ de blessures provoquées par le froid (oĩ $\dot{\varepsilon} \pi i$ $\tau \rho \omega \dot{\omega} \mu \alpha \sigma \iota ~ \kappa \alpha \tau \varepsilon \psi v \gamma \mu \dot{\varepsilon} v o เ \sigma \iota \nu$ ) ou excessivement douloureuses (ป் $\varepsilon$ p $\omega \delta \dot{v} v 0 เ \sigma \mathrm{l})$; ce sont des cas d'insensibilité

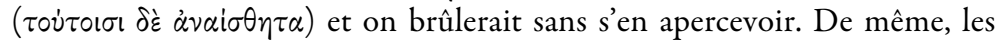
luxations profondes et considérables; on a déjà vu des pieds gelés se détacher suite à des affusions d'eau chaude. Dans ces cas, c'est la peau de celui

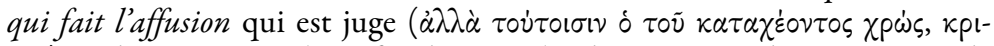
$\tau \dot{\tau}$ )), et de même pour l'eau froide. Dans les deux cas, peu d'eau n'a guère de

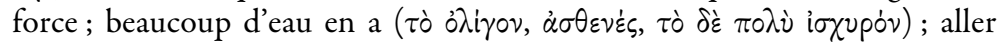

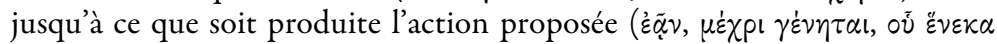

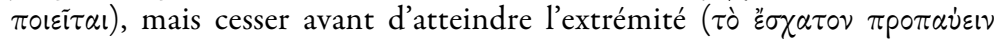
$\pi$ iv $\gamma \varepsilon v \varepsilon \dot{\varepsilon} \sigma \alpha l)$.

Chacun des deux agents provoque des dommages. [...]. La mesure dans

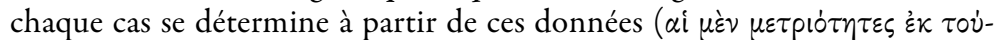
$\tau \omega \nu)$. En général, les traitements susdits sont nuisibles ou utiles ( $\beta \lambda \dot{\alpha} \pi \tau \varepsilon \mathrm{k} \kappa \alpha i$

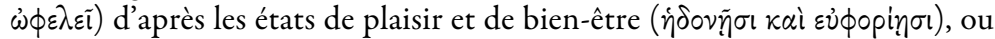
de souffrance et de malaise ( $\dot{\alpha} \chi \theta \eta \dot{\delta} \delta \sigma^{\prime}$ kai $\left.\delta \nu \sigma \phi o p i \eta \sigma \iota v\right)$, lesquels paraissent

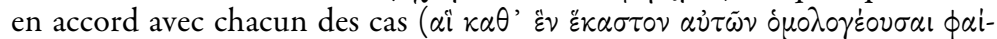

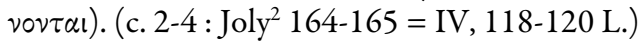

De ce texte difficile, on peut d'abord tirer une confirmation de ce que

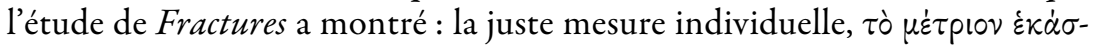
$\tau \omega$, est comprise comme un point critique entre excès et défaut. En l'occurrence, le point critique est situé sur deux échelles dont l'articulation n'est pas précisée : d'une part, celle qui va du plus froid au plus chaud, d'autre part, celle de la quantité d'eau. Il semble que l'eau n'est que le vecteur des principes actifs que sont le froid et le chaud. Si l'échelle de l'eau est proprement quantitative, il n'en va pas de même pour le chaud et le froid, qui sont présentés comme deux principes dont chacun provoque des effets qui lui

2. Joly 1972. Je modifie légèrement le texte pour les derniers mots (je ne suis pas la correction de Thivel), et modifie la traduction. 
sont propres. La gradation consiste alors plutôt en un mélange de ces deux principes qui se compensent mutuellement. Ce qui est dit des effets de chacun semble en accord avec les principes généralement admis dans les textes contemporains de la Collection hippocratique: le chaud amollit et rend fluide, le froid raidit et fige.

Pour obtenir le maximum d'utilité de l'affusion, le médecin doit aller jusqu'au point critique, sans le dépasser. On pourrait penser que l'absence d'indication du degré de chaud ou de froid approprié provient de l'absence d'instrument permettant sa mesure. Mais l'absence conjointe de toute mention d'une quantité d'eau, alors qu'une telle mesure serait facile pour un médecin grec, interdit cette explication. En fait, si le médecin ne peut exprimer le point critique par une mesure quantitative, ce n'est pas par manque d'instrument de mesure, mais parce que cette juste mesure est différente pour chaque cas singulier. C'est pourquoi la sensation du malade est seule juge, en première instance, de ce point critique. Parce qu'elle est susceptible de prendre la forme de la douleur et du plaisir, elle équivaut à un jugement normatif qui indique au médecin l'exact degré de chaleur ou de froid et l'exacte quantité d'eau chaude ou froide supportables sans nuire au corps du patient. Nuisance ou utilité trouvent ainsi leur expression immédiate dans la souffrance ou le bien-être, ou plutôt dans le caractère supportable ou insupportable de la souffrance causée par l'affusion.

Ce texte apporte en outre un témoignage sur l'évaluation, par le médecin, de la sensation exprimée par le patient, et sur l'opération effectuée par le médecin lorsque les indications du malade ne sont pas fiables.

Cette critique repose sur l'énumération de certains cas exceptionnels, dans lesquels l'expression de la sensation par le malade ne peut guider le médecin : aphonie, paralysie, comas, blessures par le froid ou trop douloureuses. On pourrait penser que, dans les deux premiers cas, le malade est incapable d'exprimer la sensation, tandis que dans les trois derniers, il ne

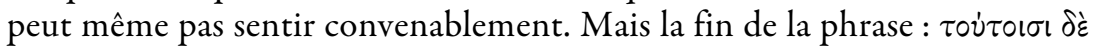
¿े $\alpha i \sigma \theta \eta \tau \alpha$, ne semble pas distinguer parmi ces pathologies. Par ailleurs, on sait que la parole peut être comprise comme l'un des sens - c'est notamment le cas dans le traité $D u$ régime (I, c. 23 : Joly ${ }^{3}, 18=$ VI, 494 L.). C'est pourquoi il faut, semble-t-il, rapporter les « anesthésies » à chacune des cinq causes énumérées. Cela conduit à comprendre la sensibilité de façon assez large, comme capacité de réagir à une altération du corps, capacité qui peut être corrompue tant au moment de la réception qu'au moment de la réaction. 
L'insensibilité par le froid peut s'expliquer par l'immobilisation des humeurs qui véhiculent la sensation. Quant à l'insensibilité par la douleur excessive, elle peut être rapprochée de l'Aphorisme II, 46: «Quand deux douleurs se produisent simultanément, mais non au même endroit, la plus forte éclipse l'autre. » (IV, $482 \mathrm{~L} .{ }^{4}$.) Il apparaît ainsi que les sensations se mélangent de telle sorte que la plus forte peut dominer le mélange au point de rendre les autres imperceptibles.

L'incapacité du patient de réagir à une altération du corps, en quoi consiste l'insensibilité, conduit le médecin à substituer le jugement de sa propre sensation à celui de la sensation du patient. La possibilité de cette substitution suppose une certaine communauté des sensibilités : la douleur ressentie par la peau du médecin peut tenir lieu de critère de la nocivité probable de l'affusion d'eau trop chaude ou trop froide, ou trop abondante, sur le corps de son malade. Toutefois, le fait que cette procédure n'intervient qu'en second lieu, dans les cas exceptionnels pour lesquels le corps malade n'est pas capable de juger lui-même du point critique, doit conduire à limiter la portée de cette communauté sensible. En général, il vaut mieux, pour le médecin, se fier au jugement du malade plutôt qu'au sien propre. La sensation du médecin n'est qu'approximativement exportable pour juger de l'effet de l'affusion sur le malade.

On trouve, dans le traité Des fractures, au lieu d'une substitution de la sensation du patient par celle du médecin, une utilisation conjointe visant un jugement par congruence. Il s'agit alors de repérer le lieu précis de la fracture :

Il faut asseoir le blessé, de manière que la partie saillante de l'os fracturé soit tournée vers la plus vive des lumières qui se trouveront là ( $\pi$ pòs $\tau \grave{\eta} \nu \lambda \alpha \mu \pi \rho \circ-$ $\tau \dot{\alpha} \tau \eta \nu \tau \tilde{\omega} \nu \pi \alpha \rho \varepsilon 0 \nu \sigma \dot{\varepsilon} \omega \nu \alpha \dot{\gamma} \gamma \dot{\varepsilon} \omega \nu$ ), pour que l'opérateur pendant l'extension

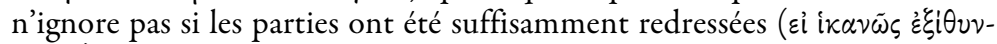

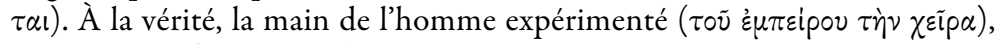

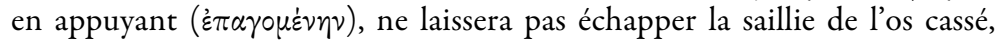
d'autant plus que c'est le point où le contact est le plus douloureux ( $2 \lambda \gamma \varepsilon \tilde{\imath}$ $\mu \dot{\alpha} \lambda 1 \sigma \tau \alpha)$. (c. 3 : Kühlewein II, 51 = III, 426 L.)

Le jugement médical résulte ici de la congruence de trois sensations : les sensations visuelles et tactiles du médecin, la sensation douloureuse du blessé. Le texte ne fait aucun départ explicite de ce qui revient au médecin et de ce qui revient au patient dans ce processus, comme si la douleur du patient était assumée par le médecin lui-même - comme si, en définitive, les deux éléments corporels en contact, la main qui parcourt le membre et le bras qui souffre de ce contact, appartenaient au même corps. 
De cet examen critique des sensations du malade, on peut conclure que l'exactitude des sensations est notamment menacée par leur mélange, s'il est intempéré, mais aussi, à l'inverse, que l'un des moyens de vérifier la pertinence d'une sensation est de la rapporter à d'autres, afin de les faire converger dans un jugement congruent. La qualité du mélange, dominé abusivement par une sensation trop forte, ou, au contraire, équilibrant les sensations selon leur importance relative, semble donc déterminer la valeur du jugement. En définitive, la critique de la sensation du patient ne débouche pas sur la mise en œuvre de critères logiques, mais sur la substitution d'une sensation à une autre, ou sur la conjugaison de diverses sensations, du patient et du médecin. Le jugement médical clinique demeure sensible, sans doute parce que la sensibilité est la mieux à même de juger d'une circonstance singulière.

\section{Esthétique du jugement clinique}

La critique des sensations concerne autant celles du médecin que celles du patient. Outre l'évaluation de la capacité du médecin de percevoir et de juger correctement, sur laquelle je reviendrai plus loin, on trouve, notamment dans le traité $D$ u pronostic, une étude de la valeur du symptôme, lequel constitue le versant objectif de la perception du médecin.

Le but du jugement clinique n'est pas, en général, de rapporter la perception d'un signe clinique à l'existence d'une lésion interne, selon un procédé qui sera systématisé, bien plus tard, par la méthode anatomo-clinique. Pourtant, l'auteur du Pronostic n'ignore pas l'expression d'une lésion locale par un symptôme local ${ }^{5}$. Mais, précisément, une telle relation menace le jugement clinique, comme le montre le c. 12, concernant les urines :

Prenez garde de vous en laisser imposer par des urines semblables que pourrait fournir la vessie atteinte de quelque affection; car alors l'urine

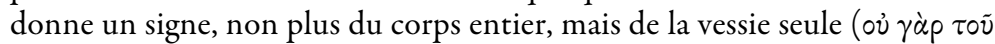

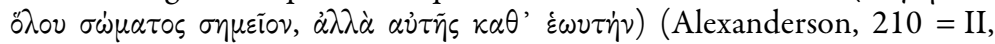
142 L. $)^{6}$.

5. La Collection hippocratique témoigne par ailleurs de la recherche d'un lien entre les symptômes apparents et les affections cachées. Le traité De l'art, notamment, affirme (c. 11) que le médecin peut, à partir de symptômes visibles, accéder à des maladies cachées au regard des yeux: «ce qui échappe au regard des yeux, tout cela est vaincu par le regard de

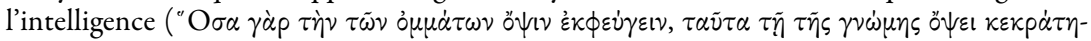
$\tau \alpha$ l). » (Texte et traduction de Jouanna 1988, p. $237=$ VI, 20 L.) Cf. Vents, c. 3 (Jouanna 1988, p. 106 = VI, 94 L.) et Régime, I, 11 (Joly 1967, p. 13 = VI, 686 L.) .

6. Pour ce traité, je suis l'édition d'Alexanderson 1963 ; je traduis (en suivant ici Littré). 
A contrario, le jugement clinique doit rassembler ce qui, des perceptions du médecin, exprime l'état du corps malade tout entier. Il doit, à cette fin, faire abstraction des symptômes d'expression locale au profit des symptômes exprimant le corps globalement. La méthode qui permet cette opération consiste à donner de l'importance aux symptômes liés entre eux. En effet, l'état général du patient est le plus souvent exprimé par un ensemble de traits convergents, tandis qu'un symptôme isolé n'exprime parfois qu'une altération locale. Le jugement clinique dont le traité $D u$ pronostic enseigne la méthode consiste donc essentiellement en un ovv$\tau \varepsilon \kappa \mu \alpha i p \varepsilon \sigma \theta \alpha$, une synthèse des observations du médecin au chevet de son malade. Charles Lichtenthaeler a désigné sous le nom de « principe de congruence » le fondement de cette opération du clinicien. Il comprend sous ce terme trois caractères propres à ce qu'il nomme le « raisonnement hippocratique $\gg$ :

— la relativité des symptômes : «Tout signe, il importe d'en évaluer la portée pronostique à la lumière de tous les autres »;

— le balancement des symptômes : «Lorsqu'un ou plusieurs signes pronostiques paraissent favorables, il faut chercher le ou les mauvais signes qui en infirment la portée, et réciproquement »;

- la complétude de l'examen : «Si nombreux et disparates que puissent être les signes pronostiques, toujours il faut les prendre tous en considération et n'en négliger aucun $\gg^{7}$.

Ces trois aspects caractérisent convenablement l'attention du praticien à la liaison des symptômes. Comme Littré le soulignait, le traité Du pronostic est un traité, non de sémiologie, mais de pathologie spéciale : il enseigne la méthode clinique dans le domaine des maladies aiguës, en vue, non d'établir des diagnostics, c'est-à-dire de subsumer les observations sous le concept d'une maladie, mais plutôt de rassembler les symptômes perçus dans une évaluation de l'état général du patient. Selon Lichtenthaeler, cette méthode ne s'oppose pas à la présence, dans le Pronostic, d'une nosologie développée par ailleurs ${ }^{8}$.

L'énoncé du principe de congruence sous la forme que lui donne Lichtenthaeler en fait un principe logique qui gouvernerait le raisonnement médical. Cette présentation tend à séparer - abusivement - d'une part l'observation des signes et d'autre part le calcul du pronostic à partir de ces observations. Le traité désigne cette opération de calcul des signes favorables et funestes par $\lambda \circ \gamma i \zeta_{\varepsilon \sigma} \theta \alpha \mathrm{l}$ ou $\sigma \nu \lambda \lambda \circ \gamma i \zeta_{\varepsilon \sigma} \theta \alpha \mathrm{l}$, termes qui apparaissent, dans ce sens, à trois reprises, une fois dans le c. 15 et deux fois dans le c. 25

7. Lichtenthaeler 1948, p. 52.

8. Voir Lichtenthaeler 1963, p. 43-101. 
qui conclut le traité. Or, l'arithmétique des signes exprimée par ce terme n'est pas distinguée de l'observation du malade. Elle ne désigne pas une opération séparée qui prendrait le relais de l'examen clinique, mais elle oriente continuellement l'observation, non seulement en dirigeant le regard du médecin vers la recherche de certains signes, mais aussi en conférant à leur manifestation une tonalité plus ou moins critique selon le jugement provisoire que le médecin a déjà ébauché en considération des signes précédents. Ainsi, le calcul est contemporain de la perception. Au rebours, la perception n'est jamais une simple observation au sens de Claude Bernard. Les verbes $\sigma \kappa \circ \pi \varepsilon \varepsilon \nu$ ou $\sigma \kappa \varepsilon \dot{\varepsilon} \tau \varepsilon \sigma \theta \alpha \iota$ et leurs dérivés désignent une investigation déjà guidée par une interrogation et comportant une dimension de

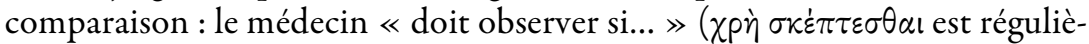
rement suivi d'une série de $\varepsilon i . .$.$) , de sorte que le regard juge en même temps$ qu'il perçoit. Il n'a pas tant pour objets des éléments anatomiques que des éléments de jugement ( $\tau \varepsilon \kappa \mu \eta p i \alpha)$ et des signes ( $\sigma \eta \mu \varepsilon i \alpha)$. L'étude lexicale ne permet donc pas de distinguer un vocabulaire de la sensibilité et un vocabulaire de la raison. Ainsi, au c. 16, le verbe $\dot{\varepsilon} \pi \iota \kappa \kappa \dot{\varepsilon} \pi \tau \varepsilon \sigma \theta \alpha \iota$ désigne la visée

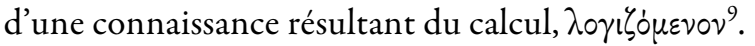

En revanche, le lexique distingue plus nettement entre d'une part le mouvement d'investigation et d'autre part la connaissance probable qui en résulte et celle sur laquelle il s'appuie. Cela ressort notamment de l'étude du c. 2, lequel décrit le syndrome de l'agonisant, resté célèbre sous le titre de « faciès hippocratique ». Dans ce chapitre, le jugement clinique associe quatre termes :

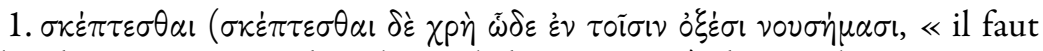
d'abord examiner ceci dans les maladies aiguës ») désigne l'investigation clinique, en l'occurrence, la recherche de signes d'abord sur le visage du ma-

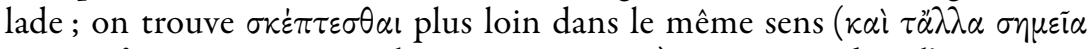
$\sigma \kappa \dot{\varepsilon} \pi \tau \varepsilon \sigma \theta \alpha$, « et examiner les autres signes 》), et $\sigma \kappa o \pi \varepsilon i \nu$ dans l'expression

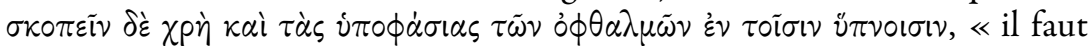
examiner aussi ce qui apparaît des yeux dans le sommeil ».

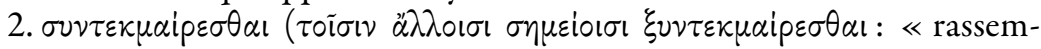
bler les autres signes en un jugement ») désigne la synthèse des « autres signes $\gg$, laquelle, quand elle est effectuée, détermine en grande part le $\sigma \kappa o \pi \varepsilon i v$ dans son objet et surtout dans sa tonalité plus ou moins pessimiste. $\sum \nu \nu \tau \varepsilon \kappa \mu \alpha i p \varepsilon \sigma \theta \alpha$, dont c'est la seule occurrence dans le traité, exprime le résultat du $\sigma \nu \lambda \lambda \circ \gamma i \zeta \varepsilon \sigma \theta \alpha$.

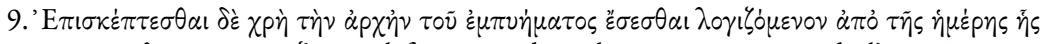

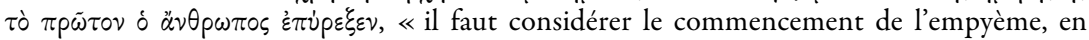
comptant à partir du jour où le malade a eu, pour la première fois, de la fièvre. » (Alexanderson, 214 = II, 150-152 L.) 


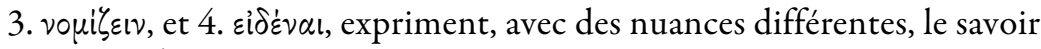

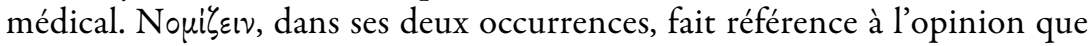
le médecin doit posséder au préalable de la valeur clinique habituelle des

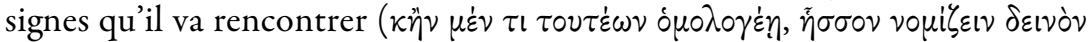

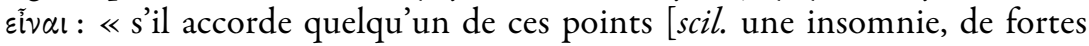
diarrhées, la faim], savoir que c'est moins funeste »; plus loin, $\tau \alpha \tilde{\nu} \tau \alpha \pi \dot{\alpha} \nu \tau \alpha$

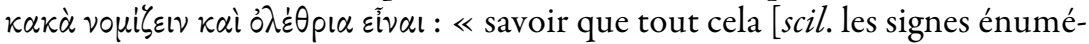
rés immédiatement avant] est mauvais et funeste $\gg)$. On retrouve voui dans le même sens aux c. 6, 12 et 13. Ce terme fait référence dans toutes ses occurrences à une vérité habituelle. Eì่̇́val ( $\theta \alpha \nu \dot{\alpha} \tau o v$ : «il faut savoir qu'il est proche de la mort »), exprime le savoir que le médecin tire de l'investigation clinique. Ce savoir n'est pas essentiellement différent d'un voir: plutôt que la conclusion d'un raisonnement, plutôt qu'une conjecture sur l'avenir, il exprime, en l'occurrence, la condamnation du malade que le regard du médecin perçoit globalement dans l'apparence présente de son patient.

Schématiquement, l'examen clinique associe donc le mouvement d'investigation au savoir habituel et au jugement des autres signes pour déterminer une perception de l'état du patient. Cette dernière se forme progressivement au cours de l'examen.

L'étude formelle de ce c. 2 du Pronostic (Alexanderson, 194-197 = II, $112-118$ L. $)^{10}$ montre que sa structure logique, imposée par la mise en forme, dans un texte, de la pratique du médecin, est débordée par la réalité qu'il décrit. Le texte construit une série de disjonctions dont chaque branche est introduite par $\varepsilon \grave{l}$ ou $\dot{\varepsilon} \dot{\alpha} \nu$, ou par une série de ces particules, pour conclure par un pronostic. Le début du texte distingue implicitement, parmi les maladies, les maladies aiguës, puis, parmi les moments de l'examen, l'examen du visage ; enfin, il oppose, parmi les apparences du visage, l'aspect de la santé et celui de l'agonie :

Voici ce que ce serait : nez effilé, yeux enfoncés, tempes affaissées, oreilles froides et contractées, et les lobes des oreilles écartés, et la peau de la région $\mathrm{du}$ front sèche, tendue tout autour, et aride. Et la couleur du visage tout entier jaune ou noire.

Le texte met ensuite en place une dichotomie : si les signes de l'agonie

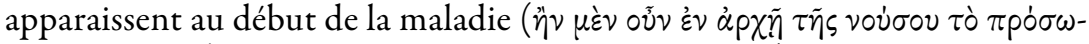

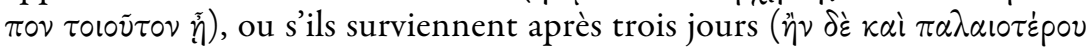

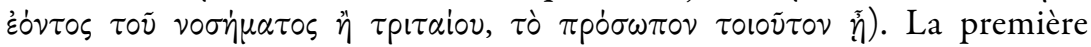
branche se subdivise selon deux cas : soit le malade déclare avoir souffert

10. Le texte de ce chapitre, établi par Alexanderson 1963, et ma traduction mot à mot en regard sont donnés en annexe. 
d'insomnie, de forte diarrhée ou de faim, soit il n'accorde aucun de ces

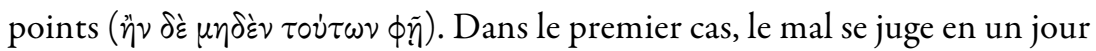
et une nuit; dans le second cas, si le syndrome ne cesse pas après ce délai, cela est signe de mort. La seconde branche de l'alternative précédente (une maladie plus ancienne) conduit à une investigation plus complexe comprenant l'interrogation et l'examen des autres signes : ceux de l'ensemble du corps et des yeux. Suit une énumération de signes funestes concernant les yeux, puis d'autres traits du visage, dont la conjonction de certains exprime l'agonie :

Si en effet les yeux fuient la lumière, ou versent des larmes, ou divergent, ou si l'un devient plus petit que l'autre, ou si le blanc de l'œil devient rouge ou si de petits vaisseaux livides ou noirs apparaissent en lui ${ }^{11}$, ou si de la chassie apparaît autour des pupilles, ou s'ils sont agités, ou exorbités, ou profondément enfoncés, ou si la couleur du visage tout entier est altérée, savoir que tout cela est mauvais et funeste. Il faut observer aussi ce qui apparaît par dessous des yeux dans le sommeil. Si en effet quelque chose apparaît du blanc, les paupières étant fermées, sans que cela vienne d'une diarrhée ou de la prise d'une potion, ou sans habitude de dormir ainsi, le signe est fâcheux et rigoureusement signe de mort. Si par ailleurs la paupière se retourne, ou devient livide, ou la lèvre, ou le nez, avec quelqu'un des autres signes, il faut savoir qu'il est sur le point de mourir.

Malgré son aspect conclusif, ce chapitre ne contient pas toutes les données nécessaires pour pronostiquer. Ainsi, il fait appel à l'examen des signes

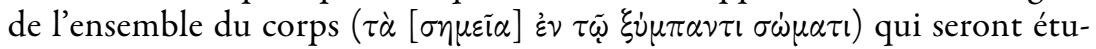
diés plus loin dans le traité. Par ailleurs, l'absence apparente d'ordre dans l'énumération des signes funestes concernant les yeux laisse penser que cette liste n'est pas close.

Le texte laisse également des ouvertures entre les branches des alternatives apparentes. Ce qui est présenté comme alternatives renvoie en fait à des séries continues dont le texte ne décrit que les deux extrêmes. Ainsi, la première distinction, entre le visage de la santé et celui de l'agonie, met en place une opposition de contraires plutôt qu'un choix entre contradictoires

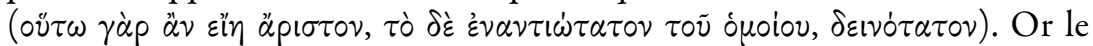
texte ignore les cas intermédiaires. Il suppose un faciès hippocratique avéré pour établir trois pronostics en considérant des combinaisons de signes funestes et de circonstances amendantes : si le faciès hippocratique apparaît au début de la maladie et qu'il y ait eu, aux dires du patient, insomnie, diarrhée ou faim, le cas est moins grave et se juge en un jour et une nuit

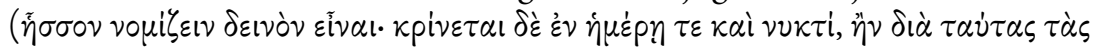

11. Ou : « le blanc devient rouge ou livide ou de petits vaisseaux noirs y apparaissent ». 


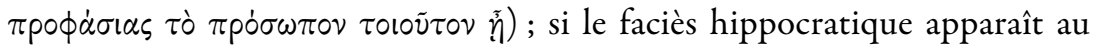
début de la maladie, mais sans aucune de ces trois circonstances, et qu'il ne

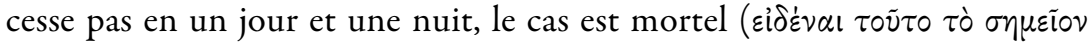
$\theta \alpha \nu \alpha \tau \tilde{\omega} \delta \varepsilon \varsigma$ ह̇ov). Si, enfin, le syndrome survient plus tard, qu'aucune des circonstances précitées, semble-t-il, n’amende le cas, et que les autres signes

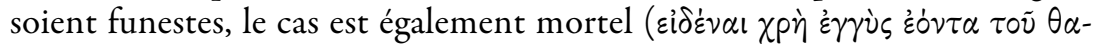
$\nu \dot{\alpha} \tau \circ v)$. Le texte laisse pourtant entrevoir d'autres possibilités. Par exemple,

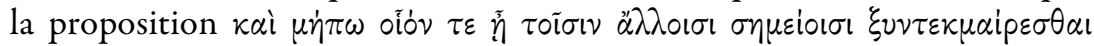
(« et qu'on ne soit pas encore capable de rassembler les autres signes en un jugement ») laisse penser qu'un examen des autres signes peut se révéler utile même dans le cas d'un syndrome précoce, en sorte qu'on peut concevoir un faciès hippocratique survenant en début de maladie, avec ou sans circonstances amendantes, associé à d'autres signes funestes; de même

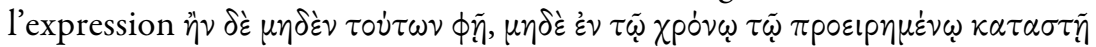
(«s'il dit non sur ces points, et que dans le temps indiqué cela ne passe pas ») ne permet pas d'exclure le cas d'une réponse négative concernant les circonstances amendantes, avec cependant une disparition du syndrome au bout d'un jour et une nuit, ou, à l'inverse, une réponse positive et un syn-

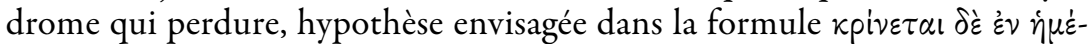

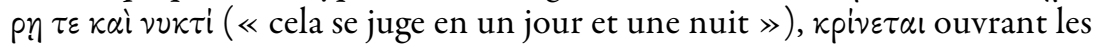
possibilités d'une issue heureuse ou défavorable, sans que les termes exacts de ce jugement de la maladie soient établis. Par ailleurs, la conjonction d'un syndrome tardif et de circonstances amendantes est envisagée, puisqu'il est prescrit, dans le cas d'un syndrome tardif, de poser les mêmes questions que dans le cas d'un syndrome précoce. Mais cette hypothèse ne donne lieu à aucun pronostic. Enfin, l'hypothèse de l'absence complète d'autres signes funestes n'est pas envisagée. En outre, la présence de signes incompatibles dans l'énumération des symptômes relatifs aux yeux (yeux enfoncés ou exorbités) et la série de disjonctions qui les relie montre que le médecin ne peut s'attendre à tous les reconnaître sur le même patient. On peut donc penser que son jugement sera d'autant plus sévère que les signes funestes s'accumuleront. Mais le texte substitue à cette gradation du jugement l'expression de pronostics absolus.

Le texte semble donc traduire et schématiser selon une architecture logique, dans la forme d'une combinaison de symptômes et de réponses du patient, ce qui relève, dans la pratique, d'un mélange total de ces éléments aboutissant, non à une alternative opposant pronostic favorable ou mortel, mais à une gradation du pronostic dans une échelle continue. Cette présentation qui sépare les cas extrêmes des séries continues pour bâtir des canevas est justifiée par le but didactique de ce texte. En parcourant jusqu'au bout le cheminement conduisant à certains pronostics, tout en laissant ouverts une 
multiplicité d'autres chemins, le texte enseigne une méthode que le médecin devra renouveler au chevet de chaque patient.

La dichotomie qui sépare apparemment de la façon la plus rigide deux genres de cas est celle qui distingue le syndrome survenant en début de maladie et le syndrome survenant après trois jours. Or, les opérations exigibles du médecin dans chacune de ces deux occurrences se rejoignent bizarrement. Si le syndrome survient au début de la maladie, il convient d'interroger le malade, mais cela n'exclut pas, par la suite, d'examiner les

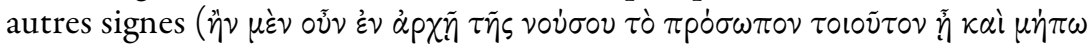

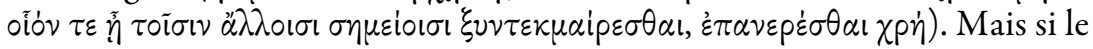
syndrome survient plus tardivement, il convient de poser les mêmes ques-

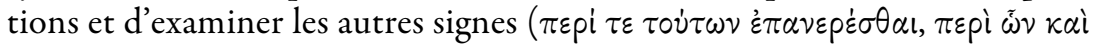

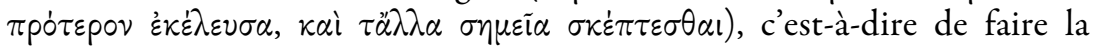
même chose ! La différence ne concerne en fait que la tonalité à la fois de l'interrogation et de l'examen. Le syndrome précoce se tranche de façon extrême. L'interrogation suffit presque à prédire soit la mort, soit la rémission. En revanche, le syndrome tardif exige une méthode plus longue. Le poids des réponses positives ou négatives du patient n'est plus déterminant; le calcul des autres signes acquiert alors plus d'importance. Cette différence de tonalité n'a pu trouver, dans la formulation écrite, qu'une expression logique trop rigide pour ce qu'il fallait exprimer, sous la forme d'une dichotomie placée, en toute rigueur, trop tôt, puisque le cheminement est similaire dans les deux branches qu'elle oppose.

C'est encore une différence de tonalité qui permet de comprendre l'apparente répétition de la considération de la couleur du visage et de l'enfoncement des yeux. Dans la première description du faciès hippocratique, ces symptômes sont en effet déjà envisagés : ỏ $\phi \theta \alpha \lambda \mu \circ \grave{~ \kappa o i ̂ ̀ o l ~[. . .] ~ \kappa \alpha i ̀ ~}$

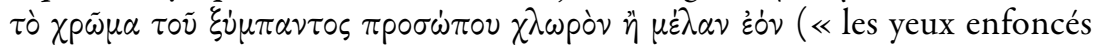
[...] et la couleur du visage tout entier jaune ou noire »). Or, on les retrouve

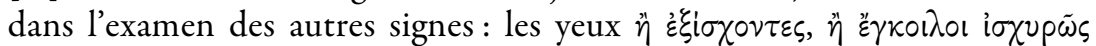
$\gamma เ v o ́ \mu \varepsilon v \mathrm{ol}^{12}$ («ou exorbités, ou profondément enfoncés »), et, concernant la

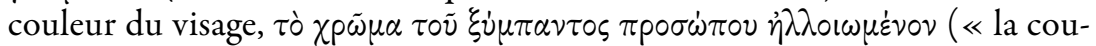
leur du visage tout entier altérée $\gg)$. Il faut considérer que le regard du praticien revient sur la couleur du visage et sur l'enfoncement des yeux avec une acuité plus grande. Mais alors, il faut conclure que le temps de ce texte n'est pas seulement le temps logique du raisonnement ou de la classification, mais d'abord le temps narratif de l'histoire de la consultation. Le

12. On peut remarquer que deux signes opposés (les yeux exorbités et les yeux enfoncés) vont dans le sens d'un même pronostic. 
médecin s'approche du patient, considère son visage globalement, puis revient sur les détails et les rapporte à ce qu'il perçoit du corps du patient.

Par ailleurs, l'arbre dichotomique laisse transparaittre une gradation des pronostics, comme si le texte appliquait sur une évolution continue du jugement un schéma logique peu approprié. Les aboutissements de chaque branche expriment des pronostics qui se situent entre les deux contraires

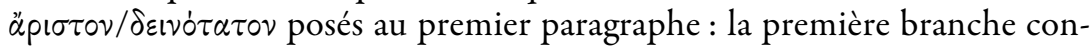
duit à juger le cas $\tilde{\eta} \sigma \sigma o \nu \delta \varepsilon เ v o ̛ v$; la seconde à considérer que les signes présa-

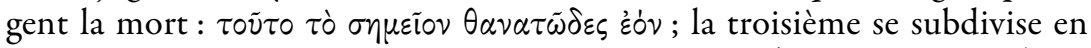

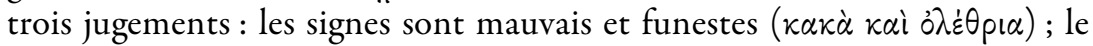
signe est rigoureusement signe de mort ( $\theta \alpha \nu \alpha \tau \tilde{\omega} \delta \varepsilon \varsigma \sigma \phi o ́ \delta \rho \alpha)$; le malade est

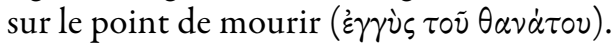

\begin{tabular}{|c|c|c|c|c|c|c|c|c|c|c|c|c|}
\hline $\begin{array}{l}\text { apparition } \\
\mathrm{du} \\
\text { syndrome }\end{array}$ & \multicolumn{8}{|c|}{ tôt } & \multicolumn{4}{|c|}{ tard } \\
\hline $\begin{array}{c}\text { circons- } \\
\text { tances } \\
\text { amendantes }\end{array}$ & \multicolumn{4}{|c|}{ non } & \multicolumn{4}{|c|}{ oui $=1$} & \multicolumn{2}{|c|}{ non } & \multicolumn{2}{|c|}{ oui } \\
\hline $\begin{array}{c}\text { état après } \\
\text { un jour et } \\
\text { une nuit }\end{array}$ & \multicolumn{2}{|c|}{$\begin{array}{l}\text { persiste } \\
=2\end{array}$} & \multicolumn{2}{|c|}{ cesse } & \multicolumn{2}{|c|}{ persiste } & \multicolumn{2}{|c|}{ cesse } & \multirow{2}{*}{ oui $=3$} & \multirow{2}{*}{ non } & \multirow{2}{*}{ oui } & \multirow{2}{*}{ non } \\
\hline $\begin{array}{c}\text { autres } \\
\text { signes } \\
\text { funestes }\end{array}$ & oui & non & oui & non & oui & non & oui & non & & & & \\
\hline
\end{tabular}

En gras : éléments donnant lieu à pronostic.

1. Syndrome précoce, avec circonstance amendante : moins grave.

2. Syndrome précoce, sans circonstance amendante, ne cessant pas après un jour et une nuit : mortel.

3. Syndrome tardif, associé à d'autres signes funestes : mortel.

Légèrement grisé : éléments envisagés sans déboucher sur un pronostic.

Syndrome précoce : le jugement en un jour et une nuit, l'examen des autres signes.

Syndrome tardif: l'interrogation sur les circonstances amendantes.

Sur fond blanc: conjonctions d'éléments rendues possibles mais non envisagées.

Syndrome précoce, sans circonstance amendante, cessant après un jour et une nuit, associé ou non à d'autres signes funestes.

Syndrome tardif sans autre signe funeste.

Enfin, un dernier trait notable de ce passage concerne les mouvements de rectification. Ils opèrent dans tous les registres : la modalité de l'observation, la valeur des signes, le jugement pronostique. 
Dès la première phrase, la modalité du $\sigma \kappa \varepsilon \dot{\varepsilon} \tau \varepsilon \sigma \theta \alpha \iota$ est rectifiée. Il faut considérer le visage en se demandant s'il est semblable à celui des bien

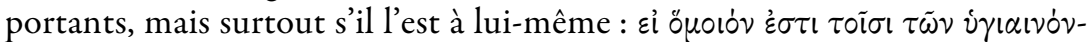
$\tau \omega \nu, \mu \dot{\alpha} \lambda \iota \sigma \tau \alpha \delta \dot{\varepsilon}, \varepsilon \dot{\imath} \alpha \dot{\nu} \tau \grave{\varepsilon} \dot{\varepsilon} \omega \nu \tau \dot{\varepsilon} \omega$. . Un critère sensible prend la place d'une idée abstraite qui ne sert que d'amorce au jugement. L'état du malade doit être jugé en référence à la santé. Mais, le médecin ne trouvant pas, dans cette idée, de critère déterminant pour le jugement, lui substitue une référence visible - plus ou moins directement, puisqu'il s'agit soit de se souvenir du visage du patient s'il est connu du médecin, soit d'interroger ses proches. La singularité de chaque conformation individuelle interdit d'abstraire un caractère universellement partagé par les visages en bonne santé. C'est pourquoi le médecin doit en définitive considérer l'égalité du visage du patient lui-même, c'est-à-dire opérer un jugement purement sensible.

La valeur des signes est elle aussi rectifiée à plusieurs reprises. Ainsi, lors de la reprise de l'examen, la couleur altérée n'est pas exactement identique à la teinte jaune ou noire évoquée au début, et, de la même manière, la saillie s'ajoute à l'enfoncement des yeux et corrige la première approche. Dans ces deux rectifications, un signe absolu (une énumération de couleurs, l'enfoncement des yeux) est remplacé par une évaluation relative à l'état antérieur de la partie (une couleur altérée, une profondeur modifiée de la situation des yeux). Par ailleurs, la référence à l'habitude du patient, ou à la prise d'une potion, amoindrit la valeur néfaste du blanc apparent des yeux, de la même manière que la veille, la diarrhée ou la faim corrigent la valeur du faciès hippocratique.

Enfin, le pronostic lui-même peut être rectifié durant l'examen. Il peut ainsi passer de $\delta \varepsilon เ \nu o ́ \tau \alpha \tau \circ \nu$, si le visage est profondément altéré, à $\tilde{\sigma} \sigma \sigma o \nu \delta \varepsilon l-$ vóv, si la vision de cette altération est compensée par l'audition des réponses positives du patient concernant les circonstances de l'apparition du symptôme.

L'absence d'ordre et de clôture des énumérations, la possibilité de cas intermédiaires dans les alternatives, l'esquisse de séries continues, les différences de tonalité et les mouvements de rectification débordent donc la structure logique de ce texte. Cet écart entre la présentation textuelle du pronostic et la pratique effective qui transparaît à travers cette présentation s'explique par la transposition dans la logique de l'écriture d'un processus qui relève du mélange des sensations. Dans la pratique, le pronostic n'est pas la conclusion d'un raisonnement construit à partir des données sensibles; il relève d'un jugement esthétique qui exprime l'équilibre mobile des perceptions qui se conjuguent, se confondent, se tempèrent mutuellement, tout au long de la consultation et du suivi médical du patient, modifiant jusqu'à l'opposer à elle-même l'impression générale qu'en tire le médecin. Le jugement ne quitte donc pas le registre de la sensibilité, comme en 
témoigne par ailleurs L'Officine du médecin (c. 1) qui énumère « ... ce qui

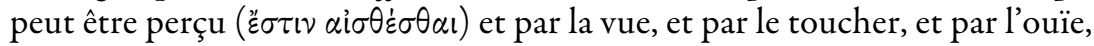

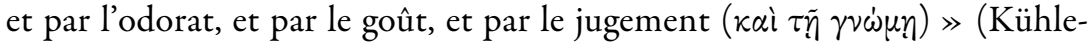
wein II, 30 = III, 272 L.), passage dans lequel, comme l'a remarqué Critias cité par Galien, la $\gamma \nu \omega \dot{\mu} \mu \eta$ est, au même titre que les sensations, objet de l'ai $\sigma \dot{\theta} \dot{\varepsilon} \sigma \alpha \iota^{13}$.

L'évocation de l'examen du malade par les Épidémies $I V$, 43, dans le désordre, l'incomplétude et l'ambiguïté d'une note elliptique, reflète bien cette opération conjointe de l'observation et du jugement chez le médecin, à quoi s'ajoute l'intrication des sensations du patient et du praticien :

[Savoir] que les jugements [se font] par les yeux, les oreilles, le nez, les

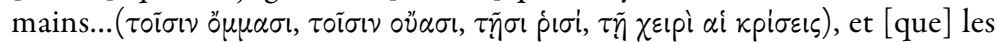

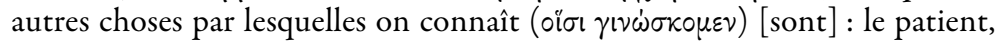
le praticien qui, en chaque cas, touche, ou sent, ou goûte, et a connaissance (yvovs) du reste: cheveux, complexion, peau, vaisseaux, tendons [autre traduction possible : nerfs], muscles, chair, os, moelle, cerveau, le sang et ses effets, intestin, estomac, bile, les autres humeurs [...] pouls, tremblements,

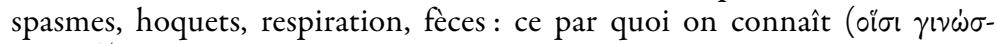

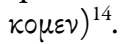

Comme le suggérait déjà Fernand Robert, il faut sans doute comprendre à la lettre l'injonction du traité Des humeurs, c. 8 (Jones ${ }^{15}, 78=\mathrm{V}, 488$ L.), lequel, après avoir énuméré certains signes que le médecin doit associer

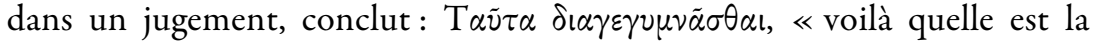
gymnastique à acquérir $\gg^{16}$.

13. Galien, In Hippocratis De Officina medici, I, 1 : Kühn XVIII B, 655, 7 = 88 B 39 D.K.

14. Je suis le texte de Langholf 1990, p. 51 note 78 (=V, 184 L.), sauf sa ponctuation, et modifie sa traduction. Je retiens notamment, pour le début du texte, la lecture difficile, mais

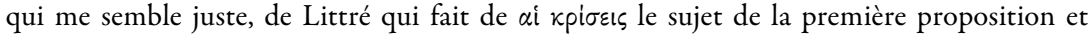
entend par ce mot le jugement du médecin (on pourrait aussi y voir les jugements des maladies en des points critiques locaux, avec la même ponctuation). V. Langholf comprend ainsi le début du paragraphe : [il faut affirmer (ou : il a été dit )] qu'[on observe] avec les yeux, les oreilles, le nez, la main. [Il y a] les crises, et les autres choses par lesquelles on fait

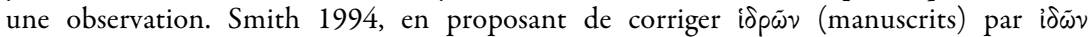
(«voyant ») plutôt que par ó $\delta \rho \tilde{\omega} \nu$ (《le praticien ») à la suite de Littré, est conduit à faire du malade lui-même celui qui a connaissance de ses symptômes : «the ill personn, seeing, touching, smelling or tasting, and knowing in the other ways » (p. 136). La suite du paragraphe énumérerait alors peut-être des éléments plus techniques, accessibles au seul médecin. La possibilité même de ces ambiguités témoigne à mon sens d'une théorie sous-jacente du jugement médical comme mélange physique de sensations du malade et du médecin.

15. Jones 1931.

16. Robert 1975. 
Dans les Épidémies VI, ce mouvement du médecin autour du malade, recueillant les éléments du pronostic, les choisissant et les mêlant selon leur valeur relative, est résumé ainsi :

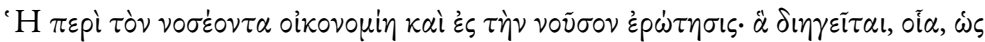

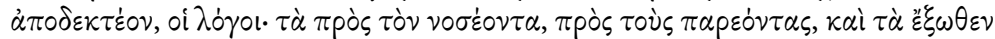
(2, 24 : Manetti-Roselli ${ }^{17}, 46=\mathrm{V}, 290$ L. $)^{18}$.

Ce texte des Épidémies VI trouve son unité si l'on y voit l'abrégé du jugement pronostique. Prenant ce parti, je proposerais la traduction suivante de ce passage, en laissant la traduction d'oikovouin et de $\lambda \dot{o} \gamma o$ en suspens :

L'économie autour du malade, et l'interrogation sur la maladie : le contenu de son discours, sa qualité, ce que cela indique. Les logoi : ce qui est relatif au malade, à l'entourage, à l'environnement.

La première phrase distingue les deux moments de l'investigation clinique : la perception des symptômes dans un mouvement d'inspection tout autour du patient, l'interrogation du malade et l'évaluation de ses propos selon leur contenu, la manière dont ils sont proférés et les indications que le médecin peut en tirer en vue d'un pronostic. Oi $\lambda$ ó $\gamma \circ$ ne désigne à mon sens ni les paroles du malade, ni celles du médecin. Si oi $\lambda$ ó $\gamma o$ s signifiait les propos du malade, l'expression serait redondante par rapport à ce qui précède. On comprendrait mal, par ailleurs, qu'elle désigne les paroles du médecin, car il serait alors fait mention d'un acte du médecin dans un contexte qui concerne l'élaboration d'un jugement sur l'état du malade; et quand bien même notre texte évoquerait ici l'activité du médecin, on ne comprendrait pas pourquoi il ne mentionnerait que la parole médicale comme activité auprès du malade. C'est pourquoi il est préférable de penser que oi $\lambda$ ó désigne des arguments qui s'ajoutent aux données tirées de l'observation directe et de l'écoute du malade. L'énumération qui suit précise donc à quoi se rapportent ces arguments : ce qui est relatif à la connaissance du malade, de son entourage, de son environnement. Cette formule est proche du premier Aphorisme qui lui aussi évoque le malade, l'entourage, l'environ-

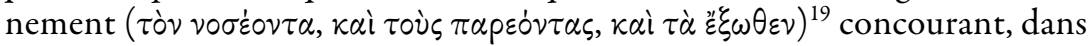
un contexte différent, à l'action thérapeutique du médecin. Le mouvement d'ensemble du texte procède par cercles concentriques, depuis la perception et l'écoute du patient jusqu'à la considération des éléments les plus extérieurs.

17. Manetti \& Roselli 1982 établissent le même texte que Littré.

18. Je remercie M. R. Alessi, qui prépare l'édition de ce texte dans la C.U.F., pour les indications très précises qu'il a bien voulu me communiquer, confirmant, dans l'état de son travail, la leçon que je suis ici.

19. Aphorismes, I, $1:$ IV, $458 \mathrm{~L}$. 
Cette occurrence unique d'oikovouin dans la Collection hippocratique mérite une attention particulière. Le contexte de la formule montre qu'il s'agit ici d'investigation clinique et non de thérapeutique, en sorte que oikovoui ne saurait désigner ici un «arrangement» actif de l'environnement domestique du patient par le médecin. Par ailleurs, le $\kappa$ ai qui suit $\dot{\eta}$

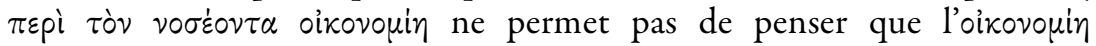
comprend les questions du médecin, puisque l'interrogation sur la maladie s'ajoute à l'économie autour du malade. Si l'interrogation s'y ajoute, l'oikovoui ne peut alors désigner que la gestion des perceptions, car perceptions et interrogation constituent la totalité de l'investigation clinique directe du patient. L'économie autour du malade désigne donc la gestion du corps du médecin autour du corps de son patient, c'est-à-dire la distribution des points de vue dans l'espace qui permet de saisir et d'assimiler au mieux les traits pertinents de l'aspect du malade et de son environnement. Si on rapporte cette formule au c. 2 du Pronostic, elle peut désigner aussi la gestion du temps de la consultation. Le va-et-vient entre la considération de l'ensemble et l'examen du détail, le retour du regard, avec une acuité plus soutenue, sur des éléments déjà envisagés, la rectification de l'impression qui se dégage au fur et à mesure des perceptions, tout cela relève en effet d'une économie du temps de la perception. Une telle gestion du temps jointe à celle de l'espace est exprimée, dans un contexte différent, par la

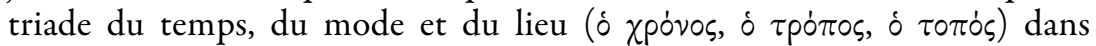
l'Officine $d u$ médecin ${ }^{20}$. S'y ajoutent peut-être les interventions des divers sens selon l'énumération des Épidémies $I V$, 43, et le produit pondéré de tous ces éléments sensibles ${ }^{21}$. Sous la forme logique défaillante d'énumérations inachevées et de dichotomies ouvertes, le c. 2 du Pronostic transcrit cette économie sensible des perceptions cliniques.

\section{Anatomie et physiologie de la sensibilité}

L'évaluation critique des perceptions concerne non seulement les symptômes perçus, mais également la capacité du médecin de percevoir et de juger correctement, comme le montre le Prorrhétique II. Ce traité pose le problème de la désobéissance du malade aux prescriptions du médecin;

20. Officine du médecin, c. 2 : Kühlewein II, $30=$ III, 76 L.

21. Galien commente Épidémies VI, 2, 24 (Wenkebach \& Pfaff 1956, p. 115-118

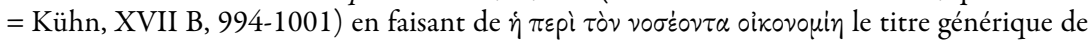
ce qui est décrit dans le texte hippocratique : l'usage des savoirs du médecin dans une appréhension globale du patient, de ses propos et de son environnement domestique. La

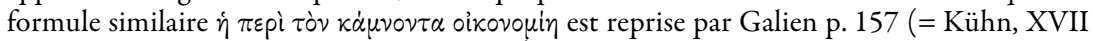
B, 63) à propos du texte hippocratique des Épidémies VI, 3, 12 (V, 299 L.). 
l'auteur se demande comment déceler les écarts de régime, tant pour l'alimentation que pour l'exercice. Ces écarts sont aisés à déceler chez le malade alité et soumis à la diète, mais plus difficiles à discerner lorsqu'on a affaire à un homme « qui va et vient et mange beaucoup ». L'auteur prescrit de visiter un tel patient chaque jour dans le même endroit, à la même heure, et surtout quand le soleil vient de se lever.

Nécessairement, c'est surtout à cette heure que l'homme vivant régulièrement se trouve, quant à la constitution de la couleur et de tout le corps,

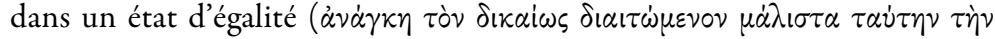

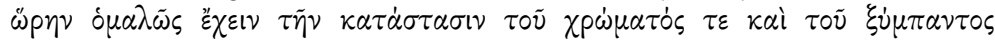
$\sigma \dot{\omega} \mu \alpha \tau o \zeta)$; et, pour la même raison aussi, c'est à ce moment que l'homme

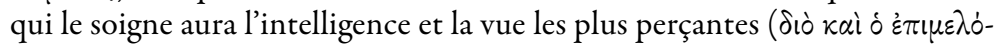

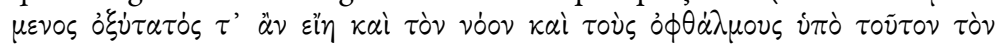
xpóvov) (c. 4 : IX, 14 L.).

Dans ce passage, l'auteur du Prorrhétique II utilise ses connaissances physiologiques pour définir les meilleures conditions de la consultation, en jugeant, d'un regard semblable, non seulement de l'état du patient, mais également de celui du médecin qui l'ausculte. Le médecin trouve alors, dans une application réflexive de la médecine, l'outil qui lui permet de juger la relation médicale elle-même. En effet, les processus de la perception et du jugement ne sont pas essentiellement différents chez le médecin et chez le patient. Ainsi, la collecte des observations par le médecin au chevet du malade et l'élaboration du jugement médical sont susceptibles d'une description biologique, au même titre, par exemple, que la description des troubles de la perception ou du jugement chez un patient. C'est pourquoi la gnoséologie qui sous-tend la pratique clinique doit s'accorder aux conceptions biologiques de la médecine hippocratique. C'est le cas dans ce passage : les mêmes raisons permettent de comprendre l'état d'égalité du corps du patient et l'acuité de la vue et de l'intelligence du médecin. Tout mouvement, toute nourriture, toute boisson modifie l'équilibre humoral et, partant, l'aspect du corps. Le bruit provoqué par ces activités empêcherait le médecin de discerner les signes de l'évolution de la maladie et ceux d'un écart éventuel de régime, si le patient n'était visité au moment où son corps est, de jour en jour, le plus égal à lui-même, car non encore troublé par les activités quotidiennes. De même, les sens et l'intelligence du médecin disposent de toute leur acuité le matin, car ils ne sont pas encore troublés par les multiples observations et jugements pratiqués dans la journée. Ces observations et ces jugements ne sont rien d'autre, du point de vue médical, que des flux d'humeurs comparables à ceux qui sont suscités par l'activité, la nourriture ou la boisson.

En raison de cette réflexivité de la médecine, les passages de la Collection hippocratique concernant l'anatomie de la perception, les pathologies senso- 
rielles et même la thérapeutique de la douleur, bien que rédigés en vue d'un traitement des malades, fournissent des éléments qui contribuent à la compréhension de la conception médicale du jugement clinique effectué par le médecin. Je me bornerai à quelques indications.

Malgré la diversité des thèses physiologiques et des descriptions anatomiques dont témoigne la Collection hippocratique, certains caractères communs peuvent être dégagés. En particulier, les traités hippocratiques considèrent en général que des fluides corporels véhiculent la sensation, l'intelligence et la motricité. Cela ressort, d'une part, des pathologies comportant une altération du mouvement du sang ou de l'air dans les vaisseaux, lesquelles impliquent une altération consécutive de la sensibilité, de l'intelligence ou de la motricité, et d'autre part de la pratique thérapeutique visant à réduire une sensation douloureuse par une interruption ou une dérivation du flux dans les vaisseaux. Par exemple, Maladie sacrée, c. 4, explique l'engourdissement [ $\nu \dot{\alpha} p \kappa \eta]$ d'une partie par une interception du flux d'air dans les vaisseaux (Jouanna ${ }^{22}, 12=$ VI, 369 L.). Parfois, le ralentissement du cours du sang est rapporté à son refroidissement, notamment par le phlegme, comme en Maladies II, c. 8, qui explique ainsi l'immobilité et la torpeur

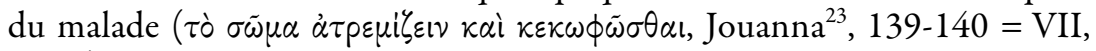
16 L.). L'arsenal thérapeutique contre la sensation de douleur comprend l'usage du chaud et du froid, de la saignée, de la cautérisation, pratiques qui visent à dériver, à ralentir ou à interrompre un flux humoral. Par exemple,

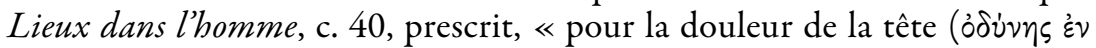
$\kappa \varepsilon \phi \alpha \lambda \tilde{\eta})$, [de] tirer du sang par les vaisseaux; si la douleur ne cesse pas et dure longtemps, cautérisez les vaisseaux, et la santé se rétablit » $\left(\mathrm{Joly}^{24}, 70\right.$ $=\mathrm{VI}, 330 \mathrm{~L}$.).

Un même usage de la saignée contre les douleurs expliquées par un rassemblement du sang est attesté par le c. 11 de Nature de l'homme $\left(\right.$ Jouanna $^{25}, 196=\mathrm{VI}, 60$ L.). Les indices d'une fonction psychique des fluides corporels sont ainsi nombreux. Maladies I va jusqu'à affirmer : « Le sang dans l'homme apporte la plus grande part de l'intelligence $(\pi \lambda \varepsilon i \tilde{\sigma} \tau \mathrm{v}$

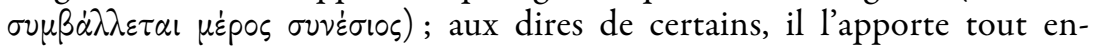
tière. » (c. $30:$ Potter $^{26}, 176-178=$ VI, 200 L. $^{27}$.)

Toutefois, à ma connaissance, aucun passage de la Collection n'attribue explicitement la transmission de la sensation aux vaisseaux. Cette impréci-

22. Jouanna 2003.

23. Jouanna 1983.

24. Joly 1978.

25. Jouanna 2002.

26. Potter 1988.

27. J'emprunte la traduction à Littré. 
sion peut en partie s'expliquer par l'absence de distinction nette entre la sensibilité, l'intelligence et la motricité, de sorte qu'il est difficile de délimiter le champ sémantique des termes désignant une faculté ou une

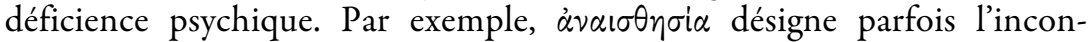
science, parfois la privation d'une sensibilité spécifique; il est difficile de savoir si $\nu \dot{\alpha} \rho \kappa \eta$, l'engourdissement, désigne seulement un trouble moteur, ou s'il s'y joint une anesthésie de la partie engourdie, et la même indécision sémantique pèse par exemple sur le sens de kwфós: muet, sourd, aveugle ou stupide. Mais l'absence d'explicitation des voies de la sensibilité a sans doute une raison plus profonde, que révèle une étude des trajets des vaisseaux.

En effet, l'étude des anatomies ${ }^{28}$ dans la Collection hippocratique ne permet de désigner aucun centre vers lequel les vaisseaux convergeraient pour transmettre des informations sensorielles. Par exemple, la description du c. 11 de Nature de l'homme (Jouanna, 192-196 = VI, 58-60 L.) reprise en Nature des os, c. 9 (Duminil, 144-146 = IX, 174-176 L.), qu'Aristote, en Histoire des animaux, III, 3, 512b11 sqq., attribue à Polybe, contient quatre paires de vaisseaux, dont la première descend de l'arrière de la tête, par le cou, les jambes et l'extérieur des chevilles vers les pieds, la deuxième descend de la région des oreilles, par les testicules, vers la face interne des pieds, la troisième, partant des tempes, forme un chiasme à hauteur des mamelles puis, par la rate ou par le foie, descend aux reins et au fondement, et la quatrième, partant des yeux, par les bras, la rate ou le foie, aboutit au pénis. La description de Nature des os, c. 8 (Duminil, 144 = IX, 174 L.), qu'Aristote, en Histoire des animaux, III, 2, 511 b23 sqq., attribue à Syennésis de Chypre, suit le trajet de deux veines qui partent des yeux pour descendre le long du dos en formant un chiasme, atteindre respectivement le foie et la rate, puis les reins et les testicules. À ces deux veines s'ajoute une nouvelle paire qui part des mamelles et se dirige vers les jambes en formant, elle aussi, un chiasme. Dans ces deux schémas, il n'est question ni du cerveau, ni du cœur, ni des phrènes. Lieux dans l'homme, c. 3 (Joly, 40-42 = VI, 280-282 L.), décrit neuf vaisseaux de la tête, qui tous partent du sinciput. Un vaisseau descend au milieu du front puis se divise en longeant chaque côté du nez, deux vont à l'angle interne de l'œil, deux passent au milieu de l'espace entre les tempes et les oreilles, deux au milieu de l'espace compris entre ces derniers et les oreilles, deux aux oreilles elles-mêmes. Par ailleurs, ce texte décrit le cheminement de deux veines qui, partant du sinciput, longent les oreilles puis se réunissent en rejoignant la « veine cave » qui descend entre la trachée et l'œsophage à travers le diaphragme et le cœur. Ce schéma ne 
permet pas de voir dans les vaisseaux des organes de transmission entre les sens et un centre. Le seul lien que Lieux dans l'homme établit entre le cerveau et un organe sensoriel par l'intermédiaire des vaisseaux concerne, au c. 2 (Joly, 40 = VI, 278 L.), les yeux. Mais alors le flux s'effectue de l'encéphale vers les yeux et sert à humecter la pupille. Maladie sacrée n'établit pas non plus un lien direct entre le cerveau et les organes sensoriels. En décrivant le trajet de chacune des deux grosses veines qui montent depuis les pieds, le traité achève sa description ainsi :

Juste auprès de l'oreille elle se cache et se divise en cet endroit là : l'embranchement le plus gros, le plus grand et le plus creux aboutit au cerveau, un autre à l'oreille droite, un autre à l'œil droit, un autre à la narine. (c. 3 : Jouanna, 12 = VI, 366 L.)

Une telle division met bien en communication l'oreille, l'œil, le nez et le cerveau, mais ne fait pas du cerveau un centre de convergence.

Ainsi, même quand elles évoquent certains organes sensoriels et certains organes qui, tels le cœur ou le cerveau, pourraient prétendre à un rôle de centralisation des signaux sensoriels, les anatomies des vaisseaux ne sont manifestement pas construites pour justifier la transmission des informations depuis les sens jusqu'à un centre de convergence qui serait le siège du sens commun ou du jugement. Au contraire, le rôle de l'appareil vasculaire est de servir de support anatomique à la thèse selon laquelle, dans le corps, tout communique avec tout. Plusieurs descriptions anatomiques tirées de cette compilation que constitue Nature des os vont dans ce sens, notamment ce passage du c. 11 (= début de Vaisseaux selon Galien ${ }^{29}$ ), qui affirme :

Les vaisseaux qui se répandent à travers le corps lui donnent souffle, flux et

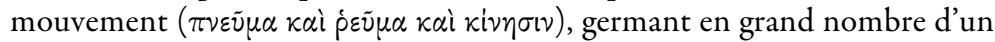

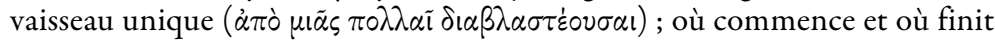
ce vaisseau unique, je ne le sais, car dans un cercle on ne peut trouver de

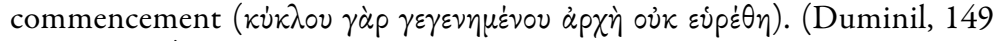
$=$ IX, $182 \mathrm{~L}$.)

Une thèse similaire est défendue par Lieux dans l'homme: « Toutes les

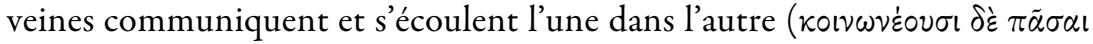

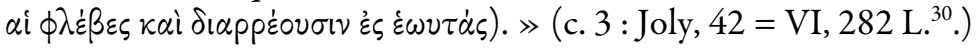

29. Kühn, XIX, 128 (le traité est mentionné sous ce titre, comme « complément au Mochlique », dans le glossaire établi par Galien des termes hippocratiques, s.v. $\pi \alpha p \alpha \sigma \tau \dot{\alpha} \sigma \alpha \varsigma)$. Voir Duminil 1983, p. 86 et sa notice de 1998, p. 75 sqq.

30. Similarité, aussi, du principe de circularité, affirmé dès l'incipit de Lieux dans l'homme: « À mon avis, rien dans le corps n'est commencement, mais tout est également commencement et fin; en effet, un cercle étant tracé, le commencement ne peut être 
De même l'angiologie du c. 9 de Nature des os ajoute aux quatre paires de veines principales d'une part « des vaisseaux nombreux et variés $(\pi \circ \lambda \lambda \alpha i$

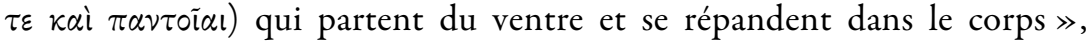
d'autre part des vaisseaux qui partent des principaux et qui communiquent

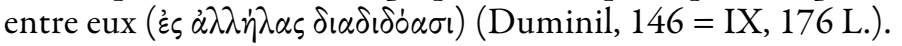

Pour autant que les fluides sont les vecteurs de la sensation ou de l'intelligence, il faut donc concevoir ces dernières comme des fonctions globales du corps, comme l'affirme Maladie sacrée: «Il existe dans le corps

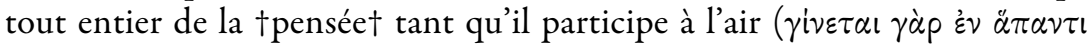

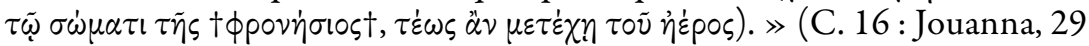
$=\mathrm{VI}, 390 \mathrm{~L}^{31}{ }^{31}$ )

C'est dans ce cadre et à cette condition que l'on peut comprendre l'affinité élective de certains lieux du corps avec la sensation, comme « la région de la poitrine $\gg$ qui, selon le c. 19 de Nature des os (=Vaisseaux), parce qu'elle retient le sang, « de tout le corps, ressent le plus les sensations

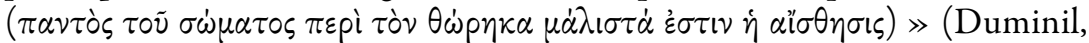
158 = IX, 196 L. $)^{32}$. Comme on le voit, la sensibilité exacerbée de la poitrine n'exclut pas celle du reste du corps. Une thèse similaire est reprise par le c. 17 de Maladie sacrée :

En provenance du corps tout entier, des vaisseaux se dirigent vers le cœur qui les maintient liés ensemble, en sorte qu'il perçoit ( $\alpha i \sigma \theta \dot{\alpha} v \varepsilon \sigma \theta \alpha \mathrm{l})$ toute

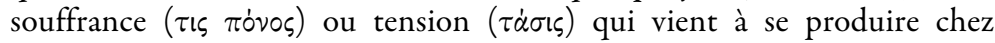
l'homme. (Jouanna, 31 = VI, 392 L.)

Toutefois, comme on le sait, Maladie sacrée attribue, non au cœur ni aux phrènes, mais au cerveau, la fonction principale en matière de pensée :

trouvé » (c. 1 : Joly 1978, 38 = VI, 276 L.), mais ce principe trouve à cet endroit une application en pathologie plutôt qu'en anatomie.

31. Je suis le texte établi par Jouanna 2003, qui comprend des améliorations notables d'un point de vue philologique par rapport aux éditions précédentes, dont certaines placent

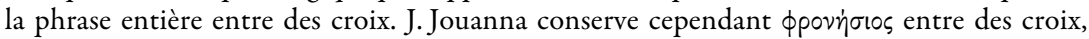
non pour de pures raisons philologiques, puisqu'il montre dans une note détaillée que le génitif partitif sujet n'est pas impossible, mais parce que «pour supprimer toute contra-

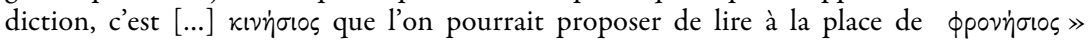
(p. 120, note 4 de la p. 29). En effet, au c. 7 il est dit que l'air fournit au corps pensée et

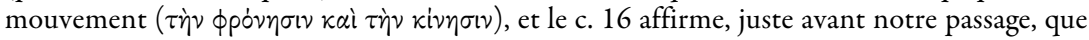

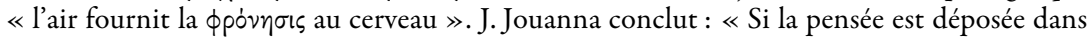
le cerveau, il reste que l'air fournit le mouvement au corps ». Pourtant, ces propositions n'impliquent pas que le cerveau soit l'organe exclusif de la pensée. De cela, on ne peut donner de meilleure preuve que cette phrase elle-même.

32. Duminil 1998 traduit : « Les sensations de tout le corps se font sentir surtout à la

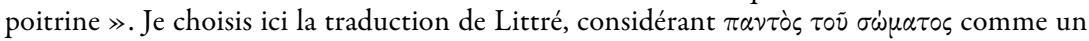
génitif partitif. 
il est le messager ( $\delta\llcorner\alpha \gamma \gamma \dot{\varepsilon} \lambda \lambda \omega \nu$, c. 16 : Jouanna, $29=\mathrm{VI}, 390$ L.) et l'interprète

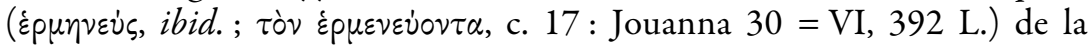

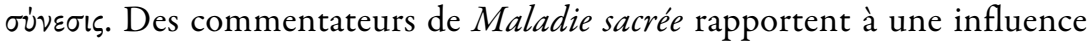
d'Alcméon ce rôle privilégié du cerveau. Selon Théophraste, en effet, Alcméon pensait que « tous les sens sont en quelque sorte suspendus (ovथnp$\tau \tilde{\eta} \sigma \theta \alpha \iota)$ au cerveau $»^{33}$. Hermann Diels ${ }^{34}$, déjà, pensait qu'Hippocrate avait tiré le rôle de l'air de Diogène d'Apollonie, cependant que l'importance du

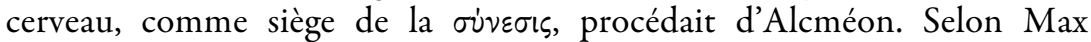
Wellman ${ }^{35}$, l'ensemble du traité serait dérivé d'Alcméon. Harold Won Miller, en 1948, en revient au partage de Diels et tente de dégager ce qui, dans le traité hippocratique, provient de chacun des deux présocratiques ${ }^{36}$. Or, cette contamination de l'interprétation du traité hippocratique par la pensée d'Alcméon suscite un malentendu concernant la fonction du

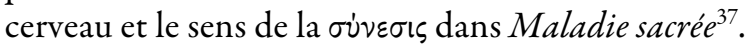

Miller est le meilleur représentant de ce malentendu, quand il déclare : «The principal thesis is unambigous: it is the brain wich, because it has received the phronêsis of pneuma, is the organic center of oiverı ; and it is the « interpreter » and the «messenger 》 to consciousness of the sensory impressions of the whole organism ${ }^{38}$. »

En réalité, le cerveau n'est nullement, pour l'auteur de Maladie sacrée, le siège d'un sens commun qui recevrait des informations en provenance des divers sens. Comme on l'a vu, le trajet des vaisseaux ne correspond pas à un tel schéma : le cerveau, s'il est irrigué par des veines en provenance de tout le corps, n'est pas un centre de convergence des voies de la sensibilité. Le

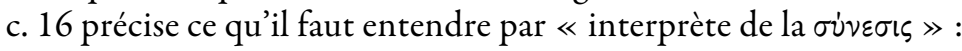

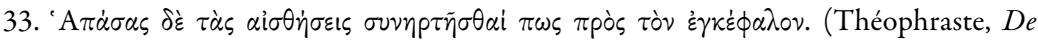
sensu, 26 = D.-K. 24 A 5.)

34. H. Diels, ap. D.-K., 6e éd., tome 2, page 68, note de la ligne 11.

35. Wellmann 1929.

36. Ce traité a fait l'objet de plusieurs études de la part de J. Pigeaud, notamment dans Pigeaud 1981, p. 33-41. J. Pigeaud fait remarquer à juste titre (p. 41) que le terme de $\psi v x \dot{n}$ n'est pas dans le texte. Il confère à l'auteur de Maladie sacrée une position «moniste ». Son interprétation demeure cependant confuse à mon sens, notamment quand il fait du cerveau « l'instrument du passage du signifiant au signifié » (p. 36). Voir aussi Pigeaud 1987, p. 5663.

37. Sur l'histoire de l'interprétation du traité, voir la notice détaillée de Jouanna 2003, notamment p. LVIII-LIX pour la question qui nous intéresse ici. Ces pages montrent bien l'effort intellectuel nécessaire pour éviter les projections rétrospectives de nos conceptions sur ce texte (voir aussi p. 121, note 5 de la p. 29).

38. Miller 1948, p. 176. 
C'est lui, en effet, qui est pour nous l'interprète de ce qui provient de l'air

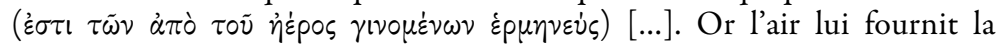

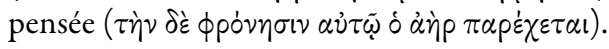

Or,

lorsque l'homme attire à lui le souffle ( $\tau \grave{o} \pi v \varepsilon \tilde{\mu} \mu \alpha)$ [par l'inspiration], le

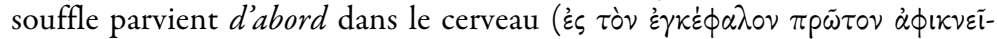
$\tau \alpha l$ ), et de la sorte, l'air se répand ( $\sigma \kappa i \delta \nu \alpha \tau \alpha l)$ dans le reste du corps (loc. cit.).

Ainsi, le cerveau ne reçoit pas les sensations pour les interpréter, il n'est pas le messager des processus corporels auprès de l'âme. Sa fonction se situe en amont : il est l'interprète et le messager, auprès du corps, de l'intelligence immanente à l'air ambiant. En diffusant l'air dans la totalité du corps, il dis-

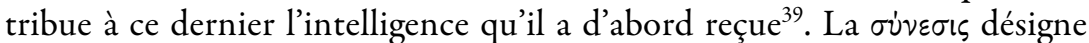
donc, non la compréhension des sensations en provenance du corps, mais la répartition de l'intelligence dans les diverses parties du corps, relativement aux circonstances. Dès lors, quand ce même texte affirme :

Les yeux, les oreilles, la langue, les mains et les pieds ne font qu'exécuter ce

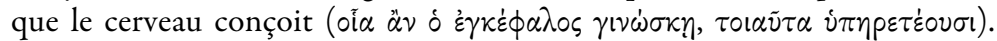

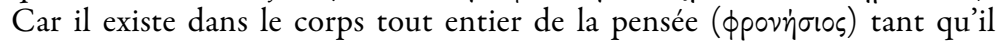

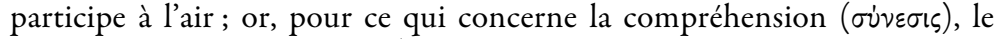
cerveau est le messager $(\text { ibid. })^{40}$,

il faut comprendre que, par son action de ventilation, le cerveau anime chaque partie du corps et permet ainsi aux yeux de voir ou aux oreilles

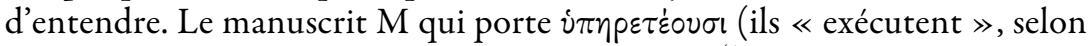
la traduction de J. Jouanna qui adopte cette leçon ${ }^{41}$ ) à la place de $\pi \rho \dot{\eta} \sigma \sigma o v \sigma \iota$

39. Une telle interprétation se heurte à l'affirmation du c. 16 selon laquelle «l'air se répand dans le reste du corps après avoir déposé [et même, déposé complètement : $\kappa \alpha \tau \alpha$ $\lambda \varepsilon \lambda o r \pi \dot{\omega} \varsigma]$ dans le cerveau ce qu'il a de plus actif en lui-même, c'est-à-dire ce qui est pensant et contient l'intelligence » (Jouanna 2003, 29 = VI, 390 L.). Cette objection est exposée par J. Jouanna dans la note 4 de la p. 29 (p. 119-121) de l'édition de la C.U.F. Mais, comme on l'a vu et comme on va le voir, plusieurs passages du traité montrent que l'auteur pense que l'air demeure chargé d'intelligence après son passage dans le cerveau, et que la différence est de degré et de priorité : le cerveau reçoit le premier l'air chargé d'intelligence, et par là même, il perçoit de façon plus précise que les autres parties du corps et est à la source de leur animation. Il reste à comprendre $\kappa \alpha \tau \alpha \lambda \varepsilon \lambda$ or $\pi \dot{\omega} \varsigma$ comme signifiant qu'aucune parcelle d'intelligence ne parvient à une partie du corps sans avoir d'abord été déposée dans le cerveau, qui est non un réceptacle, mais un relais indispensable, le « messager » de la pensée pour chaque partie du corps.

40. Traduction Jouanna légèrement modifiée : je supprime désormais les croix entou-

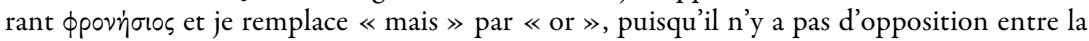
présence de la pensée dans le corps tout entier tant qu'il est ventilé par le cerveau, et l'affirmation que le cerveau est le messager de la compréhension.

41. Grensemann 1968 choisit déjà cette leçon. 
$(\Theta:$ ils «agissent »- leçon suivie par É. Littré), indique de façon plus nette encore que le rôle du cerveau se situe en amont de toute sensation. Comme dans le traité Des lieux dans l'homme, le cerveau est à l'origine, et non à l'arrivée, des flux sensoriels. Si, comme l'affirme le c. 14, c'est « de-

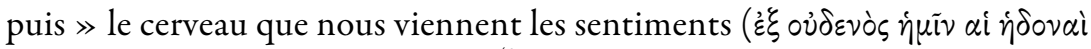

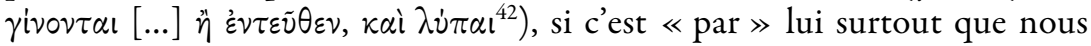

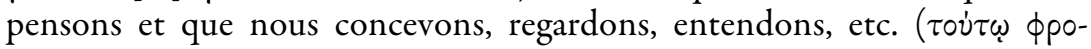

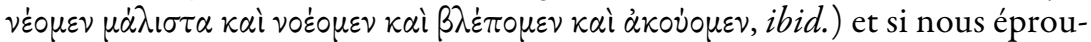

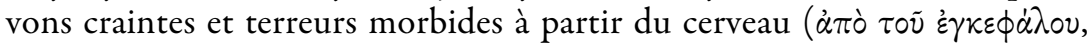
Jouanna, 26 = VI, 388 L.), cela ne signifie pas que le cerveau seul sent ou pense, mais que les fonctions psychiques de l'individu sont conditionnées par la ventilation du corps assurée par l'encéphale. Ainsi

nous devenons fous à cause de l'humidité du cerveau ; quand, en effet, il est plus humide que dans l'état naturel, nécessairement il bouge, et du fait qu'il bouge, ni la vue ni l'ouïe ne sont stables (Jouanna, 26 = VI, 388 L.).

Pour comprendre ce texte, il faut se garder d'imaginer la conscience comme un homoncule siégeant dans le cerveau, homoncule dont les perceptions seraient rendues instables par les mouvements de son support. Ce n'est pas ainsi que le médecin hippocratique se représente la pensée. L'humidité, précise le c. 13, élargit les veines : l'étiologie des troubles de la vue et de l'ouïe est mécanique. Par son mouvement, le cerveau perturbe l'aération de l'œil et de l'oreille.

La sensation et le jugement ne sont donc pas des attributs du cerveau, mais du corps tout entier, en tant que, par le cerveau, il est ventilé. C'est pourquoi, dans sa critique du prétendu rôle des phrènes ou du cœur, l'auteur conteste non leur capacité de sentir, mais la priorité de leur fonction

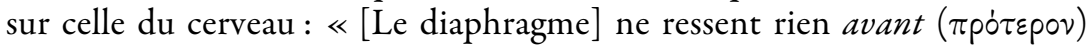
les autres parties du corps. » (c. 17 : Jouanna, 30 = VI, 392 L.) Cependant,

le cœur perçoit au plus haut point ( $\alpha i \sigma \theta \dot{\alpha} \nu \varepsilon \tau \alpha \iota \mu \dot{\alpha} \lambda ı \tau \tau \alpha)$ [l'état de chagrin ou de joie $\left.{ }^{43}\right]$, de même que le diaphragme. Cependant, aucune de ces deux

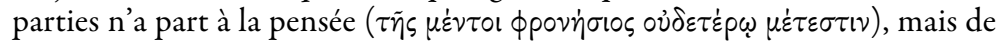

42. « La source de nos plaisirs [...] n'est autre que cet endroit-là, qui est également la source de nos chagrins [...] » (Jouanna, 25 = VI, 386 L., trad. Jouanna.)

43. Il ne convient pas de distinguer une sensibilité physique, que le traité concéderait au cœur et au diaphragme (affectés matériellement par l'état du reste du corps dans la joie ou la peine) et la sensibilité psychique (la conscience) que l'auteur réserverait au cerveau. É. Littré interprète cependant ainsi le texte, puisqu'il traduit ici $\alpha i \sigma \theta \dot{\alpha} \nu \varepsilon \tau \alpha \iota$ par «se ressentent 》.

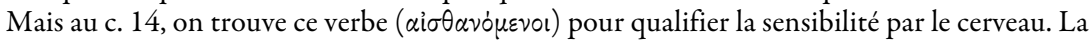
distinction dans la traduction de Littré (《se ressentent » dans un cas, « ressentant » dans l'autre) n'est donc pas justifiée. 


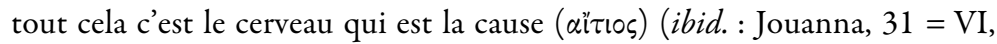
394 L.).

Le cerveau est donc la cause de la capacité des phrènes et du cœur, comme du reste du corps, d'éprouver des sensations, car quant à lui, «il

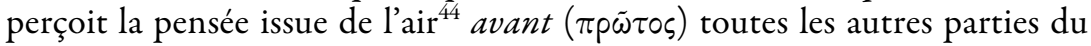
corps $\gg$ (ibid.).

Les textes hippocratiques, même lorsqu'ils font intervenir l'encéphale, ne supposent jamais une nécessaire réception par le cerveau pour qu'il y ait sensation. Ainsi, dans le c. 2 de Lieux dans l'homme, si l'audition distincte est expliquée par la pénétration du son à travers la méninge dans le cerveau,

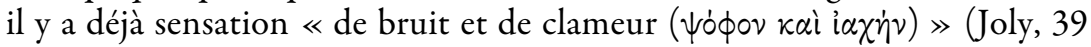
= VI, 278 L.) autour de l'oreille. Le gain de qualité peut s'expliquer, non par la nature psychophysique du cerveau, mais par la nature physique du conduit qui y mène et permet un rendu discriminant du son. On trouve d'ailleurs chez Diogène d'Apollonie une explication du même ordre du

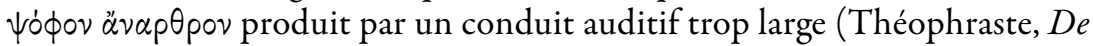
sensu, $41=64$ A 19 D.-K.) ; mais surtout, le texte même de Lieux dans l'homme confirme cette lecture, en comparant les portées respectives de l'olfaction et de l'audition, et en affirmant que l'absence d'ouverture de la méninge au niveau nasal (il y a là «quelque chose de mou comme une éponge $\gg$ ) réduit la capacité de sentir au loin.

De même, lorsque le c. 8 de Maladies II établit l'étiologie suivante d'une cécité survenant lors d'une affection, «Quant à la perte de la vision, elle est due au fait que le cerveau est situé en avant de la tête et qu'il est enflammé » (Jouanna, 139 = VII, 16 L.), la cécité n'est pas nécessairement une cécité corticale. Elle provient peut-être, comme dans Maladie sacrée, d'un effet mécanique, la partie antérieure de l'encéphale comprimant les yeux ou les vaisseaux qui y arrivent. Le c. 4 du même traité décrit un cas similaire de gonflement du cerveau dû au regorgement des petites veines périphériques, conduisant, cette fois, à une surdité :

Le cerveau remplit la cavité auriculaire avec sa propre masse et, étant donné que l'air qu'elle contient n'est plus en même quantité qu'auparavant et ne

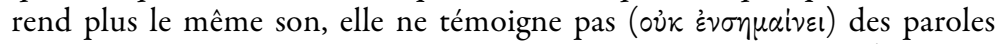
avec autant de netteté, et pour cette raison il y a dureté d'ouie (Jouanna, $135=$ VII, 12 L.).

Dès lors, ces traités de la Collection hippocratique paraissent s'accorder sur les points suivants :

1. la sensation, l'intelligence et la motricité ne sont pas nettement distinguées ;

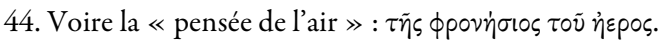


2. lorsqu'un organe comme le cerveau ou le cœur est doté d'une affinité particulière avec la sensibilité ou l'intelligence, celle-ci n'exclut pas la sensibilité des autres parties du corps ;

3. il y a sensation dès la pénétration dans le corps. La sensation n'exige pas le transfert de l'information à un centre ;

4. le rôle général des vaisseaux est de rendre compte de la solidarité de toutes les parties du corps. Dans la mesure où ils sont les vecteurs de la sensation, ils en assurent, non pas la transmission, mais la diffusion.

C'est là, me semble-t-il, la raison majeure du peu d'attention accordé aux voies de la sensibilité : la médecine ne se pose pas la question d'une conversion d'un signal physique en un phénomène psychique, et, de ce fait, elle ne désigne aucun organe pour accomplir cette fonction, ni ne cherche à poursuivre le cheminement des sensations jusqu'à ce centre. La sensation est suffisamment expliquée dès que l'on peut rendre compte d'une modification de l'état général du corps à partir de l'action d'une réalité extérieure.

\section{Conclusions}

Ces traits communs concernant les conceptions anatomiques, physiologiques et pathologiques de la sensation doivent, pour conclure, être rapportés à l'usage que le médecin lui-même fait des sensations dans sa pratique clinique. Conformément à la réflexivité de la médecine, on doit s'attendre à ce que les directives concernant les perceptions des médecins et l'élaboration de leur jugement au chevet du malade aient pour soubassement les conceptions qu'ils se forgent de la physiologie de la sensation et du jugement. Or, deux caractères dominent la formation du jugement médical : d'une part, qu'il s'agisse de pronostic ou de thérapeutique, le médecin n'applique pas une procédure fixe et universelle pour construire son jugement. Aux règles définitives établies par le raisonnement, il préfere l'attention aux sensations du patient et aux siennes propres, dont il tire une mesure singulière, qu'il renouvelle pour chaque malade, et, pour un même cas, tout au long du suivi de la maladie. D'autre part, dans l'élaboration d'un tel jugement pronostique ou thérapeutique, le médecin ne sépare pas le moment de la saisie des données sensibles de celui de leur synthèse et de leur évaluation. Le calcul des sensations n'est autre que leur tempérament, c'est-àdire leur continuelle conjugaison, leur renforcement ou leur compensation mutuels, selon leur valeur habituelle et l'état général du patient tel qu'il a pu être évalué par les perceptions précédentes. Le jugement n’est pas administré par un principe logique, fût-ce un principe de congruence. Il relève d'une économie du regard, qui, d'un même mouvement, collecte les signes, les soupèse, les balance, condamne ou délivre le malade, ou reste en suspens, dans un jugement esthétique qui n'est pas d'emblée définitif mais au 
contraire renouvelé, corrigé, ouvert, et, à ce titre, toujours plus compliqué que sa transcription littéraire.

Or, les anatomies des vaisseaux nous enseignent que les médecins hippocratiques ne traçaient pas de voies directes entre les organes des sens et un centre où siégerait le principe du jugement et de la conscience. Il y a sensation à la surface, dès le contact du chaud avec la peau, ou la pénétration du bruit dans l'oreille, c'est-à-dire dès la modification du corps par un agent extérieur. Ces sensations sont ensuite vraisemblablement véhiculées et mêlées dans les vaisseaux qui n'ont ni origine ni destination, mais qui diffusent dans le corps tout entier et modifient, selon les fluides qu'ils transportent, l'état général de l'individu. Le jugement n'est, semble-t-il, rien d'autre que cette confluence matérielle des humeurs qui véhiculent les sensations.

La maîtrise de l'art médical est donc en quelque sorte une automédication : les directives gouvernant la clinique dressent les éléments d'un régime des perceptions, excluant la considération des symptômes locaux, conseillant les heures propices à l'acuité du regard et de l'intelligence, orientant le jugement par les sens dans le temps et dans l'espace, pour susciter, par la comparaison, un mélange concordant des symptômes perçus et du savoir acquis, dans une crase sans résidu. Au régime des aliments, des exercices et des remèdes pour les malades correspond donc une économie des perceptions pour le médecin.

En guise d'épilogue, je voudrais ajouter que le soubassement physiologique de la conception du jugement médical doit être versé au dossier concernant l'énoncé du traité de l'Ancienne médecine selon lequel

il faut viser à une mesure ; or il n'y a pas de mesure - pas plus du reste qu'un nombre ni qu'un poids - à quoi l'on puisse se référer pour connaître ce qui

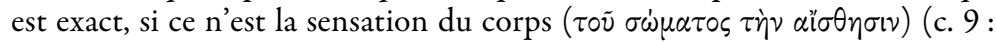
Jouanna ${ }^{45}, 128=$ I, 588-590 L.).

Ici encore, le texte avance une idée théorique, celle d'une mesure quantitative, pour lui substituer un critère sensible, la sensation du corps. Celle-ci doit être comprise dans un sens physique, comme le montre l'usage, dans le c. 15 , du terme $\alpha \nu \alpha \iota \theta \eta \eta \dot{\tau} \tau \varepsilon p \alpha$ appliqué à des réalités inanimées pour dis-

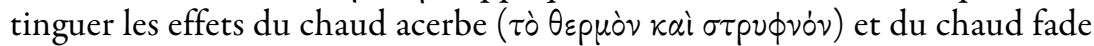

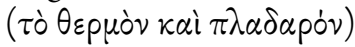

non seulement sur l'homme, mais aussi sur le cuir, et sur le bois, et sur beaucoup d'autres corps qui sont moins sensibles que l'homme (oว $\mu$ óvov $\dot{\varepsilon} \nu$

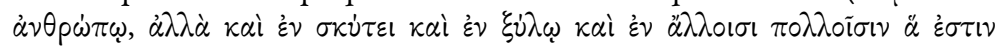
$\dot{\alpha} \nu \theta \rho \dot{\omega} \pi \circ u \dot{\alpha} \nu \alpha 1 \sigma \theta \eta \tau \dot{\tau} \tau \varepsilon p \alpha$ ) (Jouanna, $138=$ I, 606 L.). 
Cette acception physique de la sensation ne contraint pas à réduire le

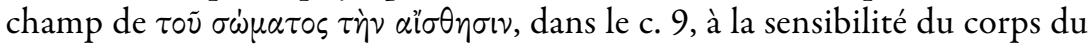
malade. Le jugement du médecin, parce qu'il a affaire à des patients toujours différents, ne peut lui-même trouver de mesure sinon dans la sensation, par son propre corps, de l'ensemble des symptômes de chaque malade. Le rassemblement, dans un jugement, de ces perceptions, est une affaire physique : affaire de mélange, dans la totalité du corps du praticien, de ce qui provient de chaque sens et de ce qu'il retient des perceptions précédentes et des valeurs habituelles de ces signes. 
ANNEXE

Pronostic, c. 2

Texte établi par B. Alexanderson, ma traduction mot à mot en face

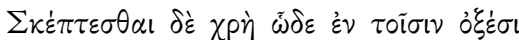

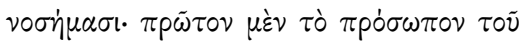

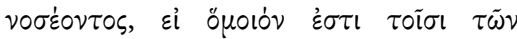

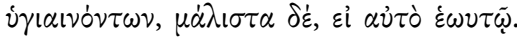

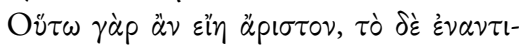
$\dot{\omega} \tau \alpha \tau \sigma \nu \tau o \tilde{u} \delta \mu \circ i o v, \delta \varepsilon เ v \dot{\tau} \tau \alpha \tau o v$.

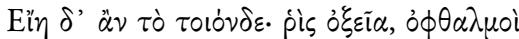
кої̀

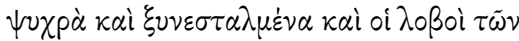

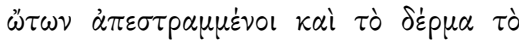

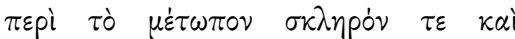

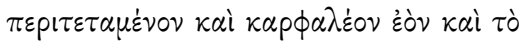

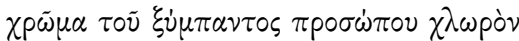
$\ddot{\eta} \mu \dot{\varepsilon} \lambda \alpha \nu$ ह่óv.

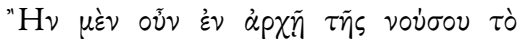

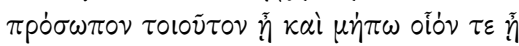

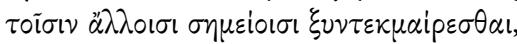

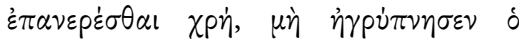

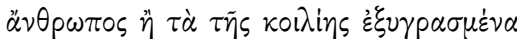

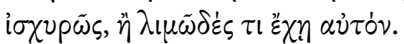

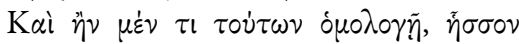

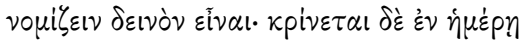

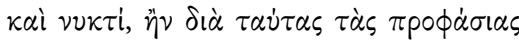

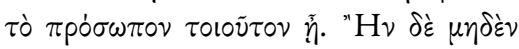

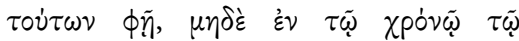

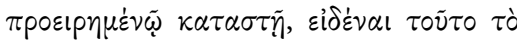

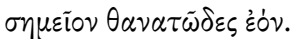

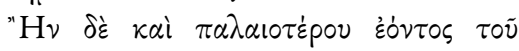

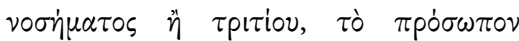

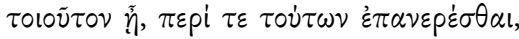

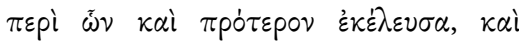

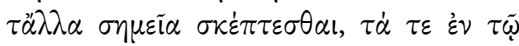

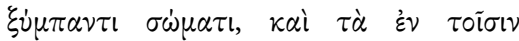
o $\phi \theta \alpha \lambda \mu \circ \tilde{\sigma} \sigma \nu$.

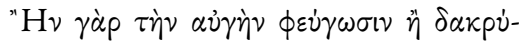

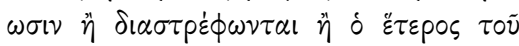

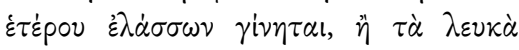

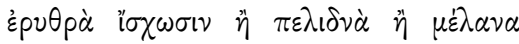

Il faut observer ceci dans les maladies aiguës : d'abord le visage du patient, s'il est semblable à ceux des bien portants, mais surtout s'il est semblable à lui-même. Ce serait le plus favorable, et le plus contraire au semblable, le plus dangereux.

Voici ce que ce serait: nez effilé, yeux enfoncés, tempes affaissées, oreilles froides et contractées, et les lobes des oreilles écartés, et la peau de la région du front sèche, tendue tout autour, et aride. Et la couleur du visage tout entier jaune ou noire.

Si au début de la maladie le visage est ainsi, et qu'on ne soit pas encore capable de rassembler les autres signes en un jugement, il faut interroger l'homme, s'il a souffert d'insomnie, de forte diarrhée ou de faim ;

et s'il accorde quelqu'un de ces points, savoir que c'est moins grave. Cela se juge en un jour et une nuit, si par ces raisons le visage est ainsi. Mais $s$ 'il dit non sur ces points, et que dans le temps indiqué cela ne cesse pas, il faut savoir que c'est signe de mort.

Si la maladie étant plus ancienne que trois jours, le visage est ainsi, interroger sur les points que $j$ 'ai dits d'abord, et considérer les autres signes, ceux du corps tout entier et ceux des yeux.

Si en effet les yeux fuient la lumière, ou versent des larmes, ou divergent, ou si l'un devient plus petit que l'autre, ou si le blanc de l'œil 


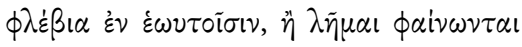

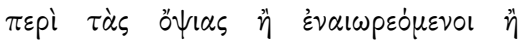

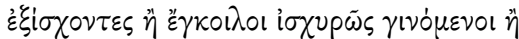

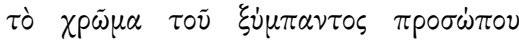
$\dot{\eta} \lambda \lambda \circ \omega \omega \mu \dot{\varepsilon} \nu \circ \nu \quad \tilde{\eta}, \quad \tau \alpha \tilde{\nu} \tau \alpha \quad \pi \dot{\alpha} \nu \tau \alpha \quad \kappa \alpha \kappa \dot{\alpha}$

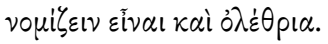

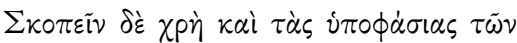

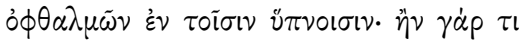

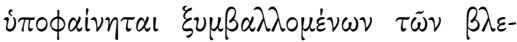

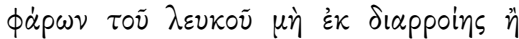

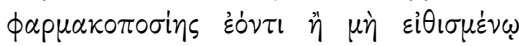

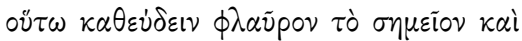
$\theta \alpha \nu \alpha \tau \tilde{\omega} \delta \varepsilon \varsigma \sigma \phi \dot{\phi} \delta \rho \alpha$.

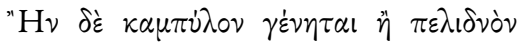

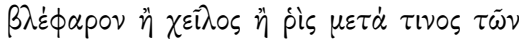

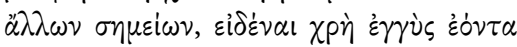

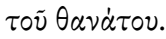

devient rouge ou si de petits vaisseaux livides ou noirs apparaissent en lui, ou si de la chassie apparaît autour des pupilles, ou s'ils sont agités, ou exorbités, ou profondément enfoncés, ou si la couleur du visage tout entier est altérée, savoir que tout cela est mauvais et funeste.

Il faut observer aussi ce qui apparaît par-dessous des yeux dans le sommeil. Si en effet quelque chose apparaît du blanc, les paupières étant fermées, sans que cela vienne d'une diarrhée ou de la prise d'une potion, ou sans habitude de dormir ainsi, le signe est fâcheux et rigoureusement signe de mort.

Si par ailleurs la paupière se retourne, ou devient livide, ou la lèvre, ou le nez, avec quelqu'un des autres signes, il faut savoir qu'il est sur le point de mourir. 


\section{BIBLIOGRAPHIE}

AleXAnderson, B. 1963 : Die hippokratische Schrift Prognostikon: Überlieferung und Text, Göteborg, 1963 (Acta Universitatis Gothoburgensis. Studia graeca et latina Gothoburgensia, 17).

Duminil, M.-P. 1983 : Le sang, les vaisseaux, le cour dans la Collection hippocratique: anatomie et physiologie, Paris, 1983 (Collection d'études anciennes).

- 1998 : Hippocrate, VIII : Plaies, Nature des os, Cour, Anatomie, texte établi et traduit par —, Paris, 1998 (Collection des Universités de France).

Grensemann, H. 1968 : Die hippokratische Schrift Über die heilige Krankheit, herausgegeben, übersetzt und erlaütert von —, Berlin, 1968 (Ars medica. II. Abteilung : griechisch-lateinische Medizin, 1).

Joly, R. 1967 : Hippocrate, VI, 1: Du régime, texte établi et trad. par —, Paris, 1967 (Collection des Universités de France).

- 1972: Hippocrate, VI, 2: Du régime des maladies aiguës; Appendice; De l'aliment; De l'usage des liquides, texte établi et traduit par —, Paris, 1972 (Collection des Universités de France).

- 1978 : Hippocrate, XIII : Des lieux dans l'homme ; Du système des glandes; Des fistules; Des hémorrö̈des; De la vision; Des chairs; De la dentition, texte établi et traduit par —, Paris, 1978 (Collection des Universités de France).

JONES, W.H.S. 1931 : Hippocrates, [Works]. 4, [Nature of Man; Regimen in Health: Humours; Aphorisms; Regimen I; Regimen II; Regimen III; Dreams]. Heracleitus, On the Universe, with an English Translation by -, Cambridge (Mass.)-Londres, 1931 (The Loeb Classical Library, 150).

JounnNA, J. 1983 : Hippocrate, X, 2 : Maladies II, texte établi et traduit par —, Paris, 1983 (Collection des Universités de France).

- 1988: Hippocrate, V, 1 : Des vents; De l'art, texte établi et traduit par —, Paris, 1988 (Collection des Universités de France).

- 1990: Hippocrate, II, 1: De l'ancienne médecine, texte établi et traduit par —, Paris, 1990 (Collection des Universités de France).

- 2002: Hippocrate, La nature de l'homme, édité, traduit et commenté par -, Berlin, ${ }^{2} 2002$ (Corpus medicorum graecorum $=$ CMG I.1.3).

- 2003: Hippocrate, II, 3: La maladie sacrée, texte établi et traduit par —, Paris, 2003 (Collection des Universités de France).

JouAnNa, J. \& C. MAgDelaine 1999: Hippocrate, L'art de la médecine, présentations, traductions, chronologie, bibliographie et notes, Paris, 1999 (GF, 838).

KÜHLEWEIN, H. 1895-1902: Hippocratis opera quae feruntur omnia, 2 vol., Leipzig, 18951902.

KÜHN, K. G. 1821-1833 : Claudii Galeni Opera omnia, editionem curavit —, Leipzig, 18211833 (Medicorum graecorum opera quae exstant, 1-20).

LANGHOLF, V. 1990 : Medical Theories in Hippocrates: Early Texts and the Epidemics, Berlin-New York, 1990 (Untersuchungen zur antiken Literatur und Geschichte, 34).

Lichtenthaeler, Ch. 1948: La Médecine hippocratique. 1, Méthode expérimentale et méthode hippocratique : étude comparée préliminaire, Lausanne, 1948.

- 1963: Quatrième série d'études hippocratiques. 7-10 [8, De l'économie du Pronostic d'Hippocrate, p. 43-101], Genève, 1963.

LITTRÉ, É. 1839-1861 : OEuvres complètes d'Hippocrate, 10 vol., Paris, 1839-1861. 
ManetTi, D. \& A. Roselli 1982 : Ippocrate, Epidemie. Libro sesto, introduzione, testo critico, commento e traduzione, Florence, 1982 (Biblioteca di studi superiori. Filosofia antica, 66).

Miller, H. W. 1948 : «A medical theory of cognition », Transactions and Proceedings of the American Philological Association, 79 (1948), p. 168-183.

PigEAUD, J. 1981 : La Maladie de l'âme: étude sur la relation de l'âme et du corps dans la tradition médico-philosophique antique, Paris, 1981 (Collection d'études anciennes).

- 1987 : Folie et cures de la folie chez les médecins de l'Antiquité gréco-romaine : la manie, Paris, 1987 (Collection d'études anciennes, 112).

POTTER, P. 1988: Hippocrates, [Works]. 5, [Affections; Diseases I; Diseases II], with an English Translation, Cambridge (Mass.)-Londres, 1988 (The Loeb Classical Library, 472).

ROBERT, F. 1975: «La prognose hippocratique dans les livres V et VII des Épidémies», dans : J. Bingen, G. Cambier \& G. Nachtergael (éd.), Le Monde grec: pensée, littérature, histoire, documents. Hommages à Claire Préaux, Bruxelles (Université libre de Bruxelles, Faculté de philosophie et lettres, 62), p. 257-270.

SMITH, W. D. 1994 : Hippocrates, [Works]. 7, [Epidemics, 2, 4-7], edited and translated by —, Cambridge (Mass.)-Londres, 1994 (The Loeb Classical Library, 477).

Wellmann, M. 1929: «Die Schrift Пepi iepñs vovंбov des Corpus Hippocraticum », Sudhoffs Archiv für Geschichte der Medizin, 22.1 (1929), p. 290-312.

Wenkebach, E. \& Fr. Pfaff 1956: Galeni in Hippocratis Epidemiarum librum VI commentaria I-VIII, ediderunt -, Berlin, 1956 (Corpus medicorum graecorum $=\mathrm{CMG}, \mathrm{V} \cdot 10.2,2)$. 